\title{
X-ray study of a sample of FR0 radio galaxies: unveiling the nature of the central engine
}

\author{
E. Torresi,${ }^{1,2 \star}$ P. Grandi, ${ }^{2}$ A. Capetti,${ }^{3}$ R. D. Baldi,${ }^{4}$ G. Giovannini,,${ }^{1,5}$ \\ ${ }^{1}$ Dipartimento di Astronomia, Università di Bologna, Via Gobetti 93/2, I-40129 Bologna, Italy \\ ${ }^{2}$ INAF-OAS Bologna, Area della Ricerca CNR, Via Gobetti 101, I-40129 Bologna, Italy \\ ${ }^{3}$ INAF-Osservatorio Astrofisico di Torino, Strada Osservatorio 20, I-10025, Pino Torinese, Italy \\ ${ }^{4}$ Department of Physics and Astronomy, The University of Southampton, SO17 1BJ, UK \\ ${ }^{5}$ INAF-IRA Bologna, Via Gobetti 101, I-40129 Bologna, Italy
}

Accepted 2018 February 22. Received 2018 February 21; in original form 2017 July 4

\begin{abstract}
FR0s are compact radio sources that represent the bulk of the Radio-Loud (RL) AGN population, but they are still poorly understood. Pilot studies on these sources have been already performed at radio and optical wavelengths: here we present the first Xray study of a sample of $19 \mathrm{FR} 0$ radio galaxies selected from the SDSS/NVSS/FIRST sample of Best \& Heckman (2012), with redshift $\leq 0.15$, radio size $\leq 10 \mathrm{kpc}$ and optically classified as low-excitation galaxies (LEG). The X-ray spectra are modeled with a power-law component absorbed by Galactic column density with, in some cases, a contribution from thermal extended gas. The X-ray photons are likely produced by the jet as attested by the observed correlation between X-ray (2-10 keV) and radio $(5 \mathrm{GHz})$ luminosities, similar to FRIs. The estimated Eddington-scaled luminosities indicate a low accretion rate. Overall, we find that the X-ray properties of FR0s are indistinguishable from those of FRIs, thus adding another similarity between AGN associated with compact and extended radio sources. A comparison between FR0s and low luminosity BL Lacs, rules out important beaming effects in the X-ray emission of the compact radio galaxies. FR0s have different X-ray properties with respect to young radio sources (e.g. GPS/CSS sources), generally characterized by higher X-ray luminosities and more complex spectra. In conclusion, the paucity of extended radio emission in FR0s is probably related to the intrinsic properties of their jets that prevent the formation of extended structures, and/or to intermittent activity of their engines.
\end{abstract}

Key words: galaxies: active - galaxies: jets - X-ray: galaxies.

\section{INTRODUCTION}

The recent wide field surveys performed in the optical and radio bands (e.g. SDSS ${ }^{1}$ and FIRST ${ }^{2}$ ) showed that the population of radio sources associated with active galactic nuclei (AGN) is dominated by objects in which the radio emission is unresolved or barely resolved in the FIRST images (e.g. Best et al. 2005; Baldi \& Capetti 2010; Baldi et al. 2018a): this implies that they have typical sizes of less than $\sim 10 \mathrm{kpc}$. In contrast, radio galaxies selected by high-flux limited lowfrequency surveys such as the 3C (Edge et al. 1959), the 3CR (Bennett 1962), the 4C (Pilkington et al. 1965) and the B2 (Colla et al. 1975; Fanti et al. 1978) often extend

* E-mail: torresi@iasfbo.inaf.it

1 Sloan Digital Sky Survey (York et al. 2000)

2 Faint Images of the Radio Sky at Twenty centimetres survey

(Becker et al. 1995; Helfand et al. 2015) to hundreds of $\mathrm{kpc}$ (FRI/FRII) and appear resolved at the angular resolution provided by FIRST. Baldi et al. (2010, 2018a) showed that the radio galaxies selected in the local Universe at $1.4 \mathrm{GHz}$, with similar bolometric luminosities, span in a broad distribution of radio luminosities and sizes, from compact to resolved with clear extended radio emission. It is important to note that a clear dichotomy is not present among sources selected from classical low-frequency radio catalogues $(\mathrm{B} 2,3 \mathrm{C}, 4 \mathrm{C})$ and radio galaxies selected in the local Universe by surveys as the FIRST.

The lack of a clear difference in luminosity and size distribution requires to adopt an arbitrary angular size threshold, that furthermore corresponds to a different physical scale depending on distance. Therefore, a precise definition of the population of compact radio sources suffers from several observational difficulties, mainly because they are selected 
from surveys (e.g. FIRST, $\mathrm{NVSS}^{3}$ and $\mathrm{AT}^{20 \mathrm{G}^{4}}$ ) limited in flux, resolution and sensitivity.

Ghisellini (2011) firstly described the compact sources studied by Baldi \& Capetti $(2009,2010)$ as FR0s. The FR0 nomenclature was then followed by Sadler et al. (2014) "as a convenient way of linking the compact radio sources seen in nearby galaxies into the canonical Fanaroff-Riley classification scheme." However, Sadler et al. found a more diversified population, e.g. with significant contribution of highexcitation galaxies (HEG), with respect to Baldi \& Capetti (2010). These differences are likely related to a substantial distinction in the luminosity functions of the two samples considered: the Sadler et al. sources extend to a radio power $\sim 100$ times higher at a radio frequency 10 times higher than the sample selected by Baldi \& Capetti.

It is also clear that compact radio sources are a very heterogeneous population and they can be produced by AGN with widely different multi-wavelength properties. For example, although most of them are radio-loud AGN, radio-quiet galaxies often show compact radio cores sometimes associated with pc/kpc scale emission (Ulvestad \& Ho 2001; Nagar et al. 2005; Baldi et al. 2018b). Furthermore, the properties of their hosts and nuclei differ depending on the frequency and flux threshold at which they are selected.

Considering the difficulties in univocally defining the class described above, Baldi \& Capetti suggested to restrict the FR0 definition to a sub-population of compact radio sources whose compactness is not due to relativistic effects and which do not follow the correlation between total and core radio power of classical FRI and FRII sources (Giovannini et al. 1988). Indeed, a source property useful to try a comparison and to select different populations with different properties could be the core dominance. Giovannini et al. (1988) discussed the core dominance properties for all sources from the $3 \mathrm{CR}$ and $\mathrm{B} 2$ catalogues with the only selection effect on the declination and galactic latitude. A clear correlation between the core and total radio power was found useful to constrain the source orientation and jet velocity. The best fit linear regression of $\log \mathrm{P}_{\mathrm{c}}$ versus $\log \mathrm{P}_{t}$ gives (see Giovannini et al. 2001):

$\log \mathrm{P}_{\mathrm{c}}=(7.6 \pm 1.1)+(0.62 \pm 0.04) \log \mathrm{P}_{\mathrm{t}}$

where $\mathrm{P}_{\mathrm{c}}$ is the core radio power at $5 \mathrm{GHz}$ and $\mathrm{P}_{\mathrm{t}}$ is the total radio power at $408 \mathrm{MHz}$.

In a pilot program of high resolution $(\sim 0$ 0.' 2$)$ radio imaging of a small sample of compact sources, Baldi et al. (2015, hereafter B15) defined as genuine FR0 those sources that appear unresolved, or slightly resolved, on a scale of $1-3 \mathrm{kpc}$ in the radio maps, that are located in red massive $\left(\sim 10^{11} \mathrm{M}_{\odot}\right)$ early-type galaxies with high black hole masses $\left(\mathrm{M}_{\mathrm{BH}} \geq 10^{8} \mathrm{M}_{\odot}\right)$ and that are spectroscopically classified in the optical as low-excitation galaxies (LEG) $)^{5}$. The sources

3 National Radio Astronomy Observatory (NRAO) Very Large Array (VLA) Sky Survey (Condon et al. 1998)

4 The Australia Telescope $20 \mathrm{GHz}$ survey (Murphy et al. 2010)

${ }^{5}$ LEG have generally weaker [OIII]-line emission with respect to high-excitation galaxies (HEG) that show [OIII]/H $\alpha>0.2$ and equivalent width of $[\mathrm{OIII}]>3 \AA$. (Laing et al. 1994; Jackson \& Rawlings 1997). More recent definitions have been provided by Kewley et al. (2006) on the basis of the of the B15 sample are highly core-dominated, since most of the emission detected at $5^{\prime \prime}$ (FIRST resolution) is included within a compact region unresolved at $45^{\prime \prime}$ (NVSS resolution): this turns out in a core-dominance higher by factor of $\sim 30$ for FR0s with respect to FRIs of the 3CR catalog.

Line luminosity is a robust proxy of the radiative power of the AGN and, at least for the sources with similar multiwavelength properties, of the accretion rate. At a given line luminosity, FR0s are $\sim 100$ less luminous than FRIs in total radio power. Therefore, the compact radio galaxies studied by B15 are not simply unresolved sources, but they show a genuine lack of extended radio emission at large scales. Possible explanations have been proposed, such as: (i) FR0s could be short-lived and/or recurrent episodes of AGN activity, not long enough for radio jets to develop at large scales (Sadler et al. 2014; Sadler 2016), or (ii) FR0s produce slow jets experiencing instabilities and entrainment in the dense interstellar medium of the host galaxy corona that causes their premature disruption (Bodo et al. 2013; B15; Baldi et al. 2018a).

In this paper we present the first systematic X-ray study of a sample of FR0 radio galaxies. Since the radio selection of compact radio galaxies carried out by Baldi \& Capetti (2010) and B15 turns out to correspond to an optical selection, we adopt these radio and spectro-photometric characteristics to define our FR0 class of low-excitation radio galaxies. This classification differs from the other FR classes, not only for the radio morphology but also for specific spectrophotometric characteristics. The key aim of our work is to investigate the central regions of FR0s through X-rays in an effort to shed light on the nature of their central engine. A comparison with the radio, optical and X-ray properties of the FRI radio galaxies is also pursued to further explore differences/similarities between these two classes of sources. Since the FR0/FRI comparison is one of the main drivers of the present study, this motivates our selection of only LEG spectroscopic types. Furthermore, this stricter definition of FR0 enables us to restrict on a more homogeneous population of compact sources, avoiding confusion with e.g. Seyfert-like objects or GPS/CSS sources (see Section 4.3). Data were taken from the public archives of the X-ray satellites currently on-flight (e.g. XMM-Newton, Chandra, Swift). Most of the X-ray data of our sample are unpublished.

Incidentally, we note that very recently a FR0 radio galaxy, i.e. Tol1326-379, has been associated for the first time with a $\gamma$-ray source (Grandi, Capetti \& Baldi 2016). Tol1326-379 shows a GeV luminosity typical of FRIs but with a steeper $\gamma$-ray spectrum that can be related to intrinsic jet properties or to a different viewing angle. For this source, a Swift Target of Opportunity (ToO) observation was performed during the writing of the paper.

The paper is organized as follows: in Section 2 we define the sample. In Section 3 we describe the observations, data reduction and spectral analysis, while the results are discussed in Section 4. Notes on single sources and details of the X-ray analysis are reported in Appendix A. The multi-

$\mathrm{L}_{[\mathrm{OIII}]} / \sigma^{4}$ quantity and Buttiglione et al. (2010) on the basis of the Excitation Index (EI) defined as $\mathrm{EI}=\log [\mathrm{OIII}] / \mathrm{H} \beta$ $1 / 3(\log [\mathrm{NII}] / \mathrm{H} \alpha+\log [\mathrm{SII}] / \mathrm{H} \alpha+\log [\mathrm{OI}] / \mathrm{H} \alpha)$. In particular, LEG sources are characterized by $\mathrm{EI} \leq 0.95$. 


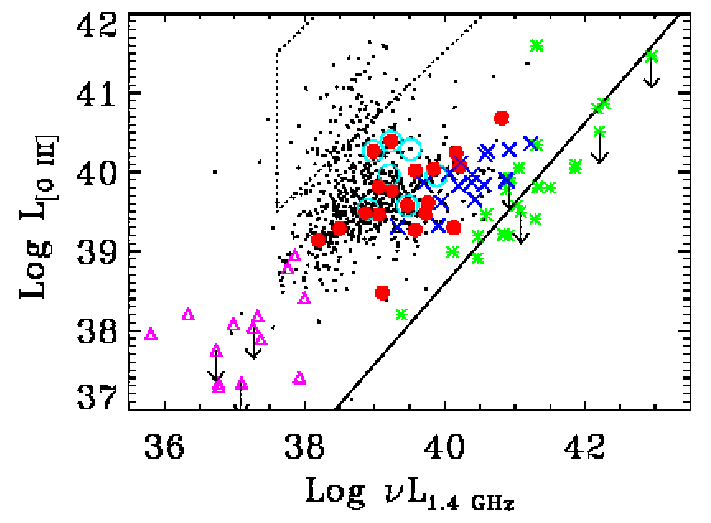

Figure 1. FIRST versus [OIII] luminosities (both in erg $\mathrm{s}^{-1}$ ) adapted from B15. Red points are the FR0s presented in this paper while empty cyan circles are the FR0s of B15. The black dot points correspond to the SDSS/NVSS sample analyzed by Baldi \& Capetti (2010), the blue crosses are the low-luminosity BL Lacs studied by Capetti \& Raiteri (2015), the empty pink triangles are the CoreG galaxies (Balmaverde \& Capetti 2006) and the green stars are the FRIs of the $3 \mathrm{CR}$ sample. The dashed line marks the boundary of the location of Seyfert galaxies. The solid line represents the line-radio correlation followed by the 3CR/FRIs.

wavelength properties of the FRI comparative sample are listed in Appendix B. Throughout the paper we use the following cosmological parameters: $\mathrm{H}_{0}=70 \mathrm{~km}^{-1} \mathrm{~s}^{-1} \mathrm{Mpc}^{-1}$, $\Omega_{m}=0.3, \Omega_{\lambda}=0.7$ (Spergel et al. 2007).

\section{SAMPLE SELECTION}

In order to build our sample of FR0 sources we took at first the SDSS/NVSS/FIRST sample of radio galaxies by Best \& Heckman (2012) ${ }^{6}$ and we applied the criteria listed below, following the approach of B15. This guarantees that we are considering LEG compact sources:

- redshift $\mathrm{z} \leq 0.15$

- compact in the FIRST images, corresponding to a radio size $\lesssim 10 \mathrm{kpc}$;

- FIRST flux >30 mJy (to ensures a higher fraction of $\mathrm{X}$-ray detected objects);

- LEG optical classification.

We obtained a list of 73 objects from which we excluded the four sources classified as low-luminosity BL Lacs (Capetti \& Raiteri 2015). We performed a search for X-ray observations of the remaining 69 sources available in the public archives of the X-ray satellites currently on-flight ${ }^{7}$ and found 15 objects. Some sources are the target of the Xray pointing, some others were serendipitous sources in the field of other targets. In order to enlarge the sample, we also

${ }^{6}$ Best \& Heckman (2012) built a sample of 18286 AGN by crosscorrelating the seventh data release of the Sloan Digital Sky Survey (SDSS) with the NRAO VLA Sky Survey (NVSS) and the Faint Images of the Radio Sky at Twenty Centimeters survey (FIRST). The sample is selected at a flux density level of $5 \mathrm{mJy}$.

7 http://heasarc.gsfc.nasa.gov/cgi-

bin/w3Browse/w3browse.pl included two FR0s already presented in B15 having public $\mathrm{X}$-ray observations. These sources were not included in our starting sample since they have FIRST fluxes $\sim 10 \mathrm{mJy}$. Finally, during the writing of the paper Tol1326-379, the first FR0 detected in $\gamma$-rays by the Fermi satellite (Grandi et al. 2016), was observed by Swift as a ToO and therefore it is considered in this work. The entire sample of FR0s studied here is reported in Table 1.

Figure 1 shows the location of our 19 FR0s in the FIRST versus [OIII] diagram adapted from B15. FR0s and $3 \mathrm{CR} / \mathrm{FRIs}$ share the same range in $\mathrm{L}_{[\mathrm{OIII}]}$, but $\mathrm{FR} 0$ s have lower radio luminosities: this strong deficit in total radio emission places FR0s to the left of 3CR/FRIs (B15), confirming that our selection criteria are valid. Even considering low-luminosity radio galaxies such as FRICAT sources (Capetti et al. 2017), FR0s still occupy the left side of the plot (see Figure 6 of Baldi et al. 2018a) forming a continuous distribution from FR0 to sFRICAT, FRICAT and 3CR/FRI sources. The low-luminosity BL Lacs of Capetti \& Raiteri (2015; see also Baldi et al. 2018b) are also shown in Figure 1 and have generally $1.4 \mathrm{GHz}$ radio luminosities higher than FR0s (see Section 4.2). In the same plot also CoreG galaxies $^{8}$ are reported (for more details see Balmaverde \& Capetti 2006; Baldi \& Capetti 2009).

The radio properties of our sample meet the FR0 criteria discussed by B15. The sources generally show flat radio spectra and are compact. Indeed, as shown in Table 2, the ratios between the FIRST and NVSS fluxes at $1.4 \mathrm{GHz}$ are around 1 , indicating that the extended component in these sources is negligible. The core dominance $R^{9}$ is on average $\sim 30$ times higher than $3 \mathrm{CR} / \mathrm{FRIs}$ and overlaps with the FR0 values of B15 (Figure 2). The paucity of information at radio frequencies higher than $1.4 \mathrm{GHz}$ for the FRICAT sources prevent us from comparing their core dominance with our $\mathrm{X}$-ray sample of FR0s. Finally, the radio spectral indices measured between $8.5 \mathrm{GHz}(4.9 \mathrm{GHz})$ and $1.4 \mathrm{GHz}$ are generally flat with a median value $\alpha_{r}=-0.04$ (see Table 2 for more details).

\section{X-RAY OBSERVATIONS AND ANALYSIS}

\subsection{Data reduction}

Data were collected from different X-ray satellites. In particular, 7 sources were observed with Chandra, 4 with $X M M$ Newton and 8 with Swift/XRT. The observation log is in Table 1. Several FR0 sources are not the primary target of the observation but are in the field of view of the pointing. The offset, i.e. the distance from the center of the source cone ${ }^{10}$ is also reported in Table 1 . When more than one

8 CoreG galaxies are low-luminosity radio sources hosted by early-type galaxies and defined "core" on the basis of the presence of a shallow core in their host surface brightness profile.

${ }_{9} R$ is defined as the the ratio between $8.5 \mathrm{GHz}$ (CLASSSCAT: Myers et al. 2003; Browne et al. 2003) and $1.4 \mathrm{GHz}$ (NVSS) flux densities.

10 See also https://heasarc.gsf c.nasa.gov/W3Browse/w3browsehelp.html\#distance_from_center 
Table 1. Log of the observations of the FR0 sample.

\begin{tabular}{lllll}
\hline \hline SDSS name & Telescope & ObsID & Exposure [ks] & Offset ['] \\
\hline J004150.47-091811.2 & Chandra & 15173 & 42.5 & 3.4 \\
J010101.12-002444.4* & Chandra & 8259 & 16.8 & 0.0 \\
J011515.78+001248.4* & XMM & 0404410201 & 54.0 & 0.095 \\
J015127.10-083019.3 & Swift & 00036976004 & 5.6 & 0.894 \\
J080624.94+172503.7 & Swift & 00085577001 & 1.3 & 0.337 \\
J092405.30+141021.5 & Chandra & 11734 & 30.1 & 0.0 \\
J093346.08+100909.0 & Swift & 00036989002 & 12.2 & 1.867 \\
J094319.15+361452.1 & Swift & 00036997001 & 5.4 & 3.437 \\
J104028.37+091057.1 & XMM & 0038540401 & 24.0 & 0.003 \\
J114232.84+262919.9 & XMM & 0556560101 & 32.9 & 13.7 \\
J115954.66+302726.9 & Swift & 00090129001 & 3.4 & 2.803 \\
J122206.54+134455.9 & Swift & 00083911002 & 1.3 & 7.641 \\
J125431.43+262040.6 & Chandra & 3074 & 5.8 & 2.0 \\
Tol1326-379 & Swift & 00034308001 & 4.3 & 0.0 \\
J135908.74+280121.3 & Chandra & 12283 & 10.1 & 3.1 \\
J153901.66+353046.0 & Swift & 00090113002 & 2.8 & 0.96 \\
J160426.51+174431.1 & Chandra & 4996 & 22.1 & 2.5 \\
J171522.97+572440.2 & Chandra & 4194 & 47.9 & 0.0 \\
J235744.10-001029.9*a & XMM & - & - & - \\
\hline
\end{tabular}

* The source is already present in the FR0 sample of B15.

${ }^{a}$ This source is part of the Third XMM-Newton Serendipitous Source Catalog, Sixth Data Release (3XMM-DR6; Rosen et al. 2016).

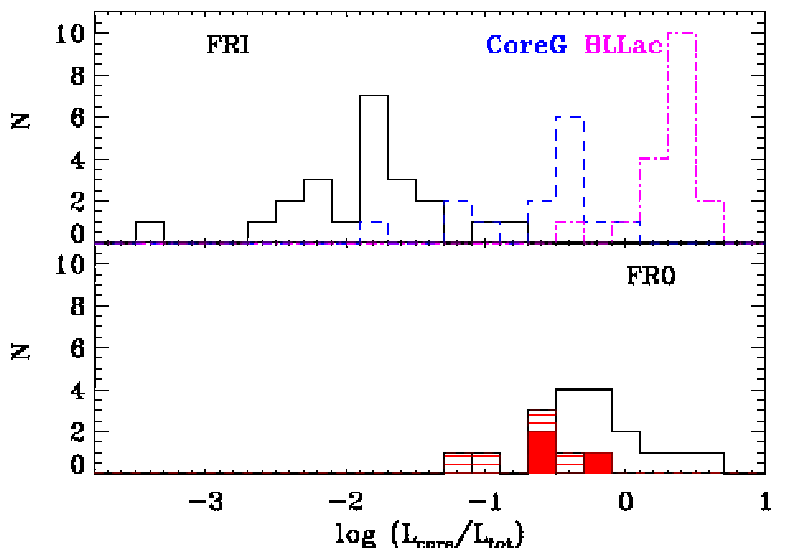

Figure 2. Histograms of the core dominance (adapted from B15) for: upper panel-3CR/FRIs (black solid line), CoreG (blue dashed line), low-luminosity BL Lacs (magenta dot-dashed line). Lower panel- FR0s of B15 (red ticked line) and our sample of FR0s (black solid line). The red filled histograms are the sources in common with B15.

observation was available, we chose the one with the smaller offset or the longer exposure. We reduced data for all the sources but one, i.e. J235744.10-00, since it is part of the 3XMM-DR6 catalog (Rosen et al. 2016).

All Chandra observations were performed using CCD, both ACIS-S and ACIS-I. Data were reprocessed using CIAO version 4.7 with calibration database CALDB version 4.6.9 and applying standard procedures. Table A1 reports the extraction regions chosen for the nuclear and background spectra of each source. Data were then grouped to a minimum of 15 counts per bin over the energy range 0.5-7 keV.
None of the seven sources pointed by Chandra is affected by pile-up.

For the XMM-Newton observations we reduced and analyzed data from the EPIC-pn camera using SAS version 14.0 and the latest calibration files. Periods of high particle background were screened by computing light curves above $10 \mathrm{keV}$. Extraction regions for the source and background spectra are reported in Table A1. Data were then grouped to a minimum of 15 counts per bin over the energy range 0.5-10 keV for two out of the three sources. For the source J235744.10-00 the 0.2-12 keV flux was directly taken from the 3XMM-DR6 catalog, and extrapolated to $2-10 \mathrm{keV}$ assuming the same spectral slope adopted in the catalog.

Swift/XRT data were reduced using the online data analysis tool provided by the ASI Space Science Data Center (SSDC) ${ }^{11}$. The only exception is Tol1326-379 that was observed as a ToO during the writing of the paper and the data were processed and analyzed using standard XRT tools (xrtpipeline v.0.13.2 and caldb v.1.0.2). Source spectra for each observation were extracted from a circular region of $20^{\prime \prime}$ radius, while the background was taken from an annulus with an inner radius of $40^{\prime \prime}$ and outer radius of $80^{\prime \prime}$. Spectra were grouped to a minimum of 5 (or 3 ) counts per bin in the energy range $0.5-10 \mathrm{keV}$. In the case of the source J153901.66+35 no grouping was applied.

\subsection{Imaging analysis}

The inspection of the X-ray images indicates that 6 sources are in a dense environment (Figure 3). Four lie at the outskirts of a cluster of galaxies (i.e. J160426.51+17, J092405.30+14, J135908.74+28,

11 http://swift.asdc.asi.it/ 
Table 2. Radio parameters: (1) source name, (2) flux ratio between FIRST and NVSS fluxes, (3) radio spectral index $\left(\mathrm{S}_{\nu} \propto \nu^{\alpha}\right)$ between $8.5 \mathrm{GHz}$ (The Cosmic Lens All Sky Survey, CLASSSCAT, Myers et al. 2003, Browne et al. 2003) and 1.4 GHz (NVSS) otherwise specified in the notes, (4) core dominance, $R$, defined as the ratio between $8.5 \mathrm{GHz}$ (CLASSSCAT) and $1.4 \mathrm{GHz}$ (NVSS) flux densities.

\begin{tabular}{lcrr}
\hline \hline Source name & $\mathrm{F}_{\text {FIRST }} / \mathrm{F}_{\text {NVSS }}$ & $\alpha_{r}$ & $\log R$ \\
\hline J004150.47-09 & 0.74 & $-0.13^{a}$ & - \\
J010101.12-00 & 0.70 & $-0.45^{b}$ & $-0.47^{b}$ \\
J011515.78+00 & 1.05 & $-0.04^{b}$ & $-0.06^{b}$ \\
J015127.10-08 & 0.88 & - & - \\
J080624.94+17 & 0.96 & - & - \\
J092405.30+14 & 0.95 & - & - \\
J093346.08+10 & 0.80 & -0.47 & -0.37 \\
J094319.15+36 & 0.99 & 0.82 & 0.65 \\
J104028.37+09 & 0.94 & $-0.63^{a}$ & - \\
J114232.84+26 & 0.94 & 0.11 & 0.09 \\
J115954.66+30 & 1.06 & -0.01 & -0.01 \\
J122206.54+13 & 0.99 & 0.28 & 0.22 \\
J125431.43+26 & 0.98 & -0.47 & -0.37 \\
Tol1326-379 & - & $0.37^{c}$ & -0.42 \\
J135908.74+28 & 1.12 & -0.12 & -0.09 \\
J153901.66+35 & 0.92 & -0.10 & -0.08 \\
J160426.51+17 & 0.75 & 0.15 & 0.12 \\
J171522.97+57 & 0.86 & -0.43 & -0.34 \\
J235744.10-00 & 0.65 & $-0.67^{b}$ & $-0.58^{b}$ \\
\hline
\end{tabular}

${ }^{a} \alpha_{r}$ between $4.9 \mathrm{GHz}$ (JVASPOL or PMN) and $1.4 \mathrm{GHz}$.

${ }^{b}$ Values of $\alpha_{r}$ and $R$ are from B15.

${ }^{c}$ The value of $\alpha_{r}$ is from Grandi et al. (2016).

J011515.78+00), J004150.47-09 is located at the centre of the cluster Abell85 and J171522.97+57 is the brightest member in a compact group of more than 13 galaxies (Pandge et al. 2012).

For the sources for which a clear extension cannot be confirmed by the X-ray images, information on the environment was checked in the literature (see Table 3). Other 4 sources were found in clusters or compact groups (CG), i.e., J093346.08+10, J122206.54+13, J080624.94+17, J115954.66+30 (Diaz-Gimenez et al. 2012; Owen et al. 1995; Koester et al. 2007). In summary, we found that at least $50 \%$ of the FR0s of our sample is in a dense environment. This value should be considered as a lower limit to the fraction of FR0s in dense environments in our sample since the analysis suffers from a bias due to the nature of the sample mainly consisting of X-ray serendipitous sources. However, we are aware that strong conclusions on the environment of FR0 as a class cannot be drawn with the available data.

\subsection{Spectral analysis}

The spectral analysis was performed using the XSPEC version 12.9 .0 package. We applied a $\chi^{2}$ statistics to spectra binned to a minimum of at least 15 counts per bin. When the grouping was smaller a C-statistics was adopted. Errors are quoted at $90 \%$ confidence for one interesting parameter $\left(\Delta \chi^{2}=2.71\right)$. The summary of the best-fit spectral results is reported in Table 3 . Notes on single sources can be found in Appendix A.

Spectral fitting was performed in the energy range 0.5-
$7 \mathrm{keV}$ (Chandra) and 0.5-10 keV (XMM-Newton and Swift). The X-ray luminosities presented throughout the paper are calculated in the $2-10 \mathrm{keV}$ range in order to make a direct comparison with the literature. As a baseline model, we considered a power-law convolved with the Galactic column density (Kalberla et al. 2005). In 4 out of 6 sources for which we could directly observe the cluster in the X-ray images (Figure 3), residuals showed evidence for the presence of a soft component. Therefore we included a thermal model (APEC). A thermal component was also required in other three FR0s. The presence of a compact group was attested in J093346.08+10 and J115954.66+30 checking the literature. For the third one (i.e J015127.10-08) no information on the environment was found. The nature of the soft X-ray emission is however uncertain. It could be due to an extended intergalactic medium (that can not be revealed because of poor X-ray spatial resolution and/or short exposure time) or related to the hot corona typical of early-type galaxies (Fabbiano, Kim \& Trinchieri 1992).

We could measure the power-law photon indices $\Gamma$ for 7 out of 18 objects. The spectral slopes are generally steep, with a mean value $\langle\Gamma\rangle=1.9$ and a standard deviation of 0.3. When it was not possible to leave the photon index free, it was fixed to a value of 2 . We checked whether different values of the photon index lead to significant changes in the estimate of the fluxes. We found that for $\Gamma$ ranging between 1.5 and 2.5 the fluxes are consistent within the errors. In four cases the low statistics did not allow us to constrain the power-law component and to exclude the presence of thermal emission, therefore we assumed a simple power-law $(\Gamma=2$ fixed) as the best-fit model and we adopted the resulting 2-10 keV flux as upper limit for the nuclear component.

Generally, the X-ray spectra of our sample do not show evidence for intrinsic absorption. Indeed, the addition of an intrinsic absorber component does not improve significantly the fit. An upper limit to this component can be estimated only for three out of 18 sources (see Appendix A). Therefore, we tend to favor the scenario in which the circum-nuclear environment of FR0 is depleted of cold matter, similarly to FRIs (Balmaverde et al. 2006; Baldi \& Capetti 2008, 2010; Hardcastle et al. 2009).

The analyzed FR0s have X-ray nuclear luminosities covering three orders of magnitude $10^{40-43} \mathrm{erg} \mathrm{s}^{-1}$. The average value including the upper limits is $\left\langle\log \mathrm{Lx}_{\mathrm{X}}>=41.30\right.$ (see Figure 4 upper panel).

\section{DISCUSSION}

\subsection{Compact versus extended low-excitation radio galaxies}

FR0/LEGs and FRI/LEGs reside in similar galaxies and share similar nuclear optical properties (B15 and references therein). Given that low-ionization optical spectra can be also produced by shocks or old stellar population emission (Binette et al. 1994; Sarzi et al. 2010; Capetti \& Baldi 2011; Balmaverde \& Capetti 2015; Mingo et al. 2016), this Xray study is a key tool to compare FR0 and FRI properties taking advantage of an energy band directly related to the nuclear emission processes.

We compared the X-ray luminosities of our sample to 

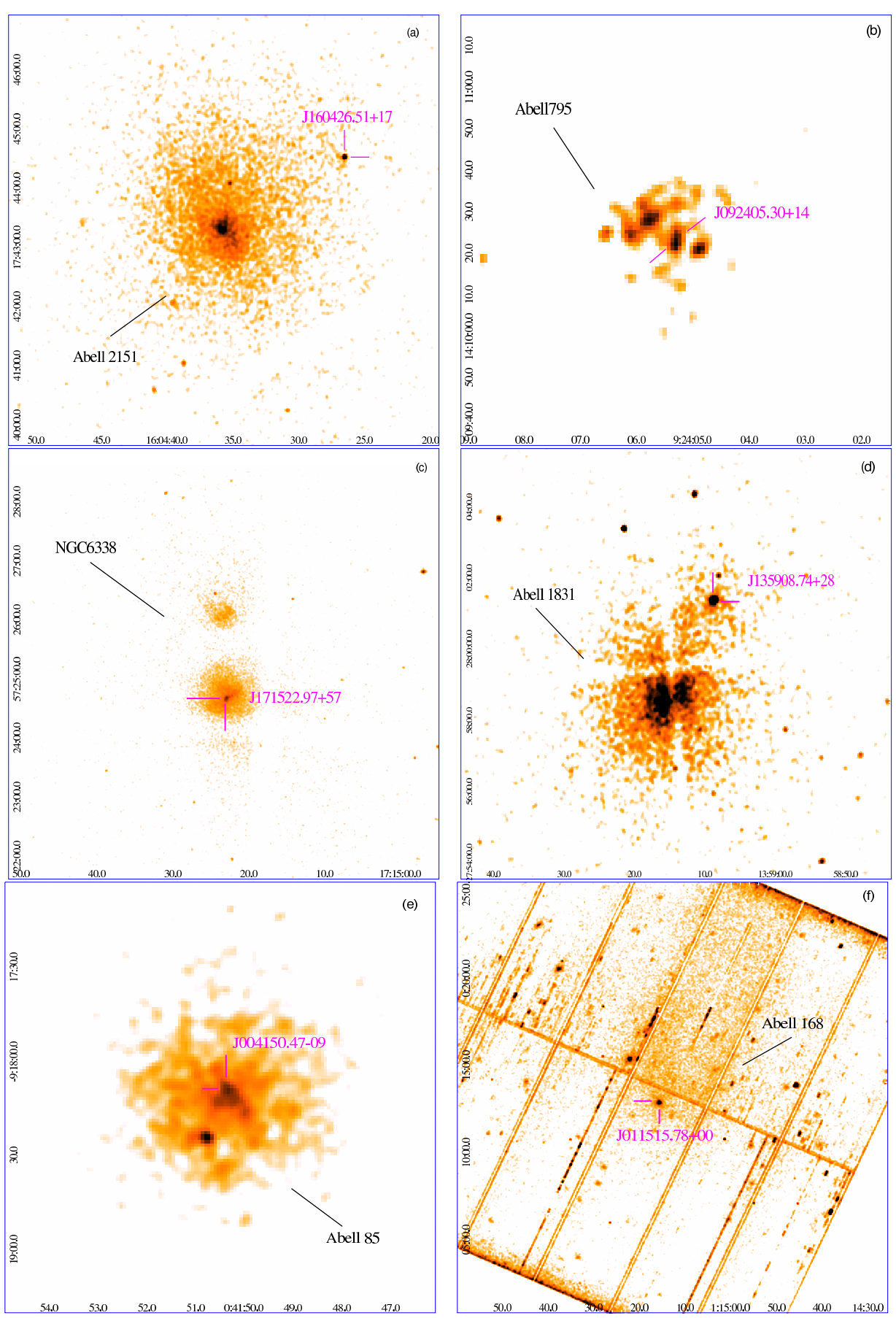

Figure 3. Images of the clusters found in the X-ray band. Panels (a)-(d): Chandra 0.3-7 keV images of the sources J160426.51+17, J092405.30+14, J171522.97+57, J135908.74+28. The FR0 is labelled in magenta while the name of the cluster is in black. Panel (e): Chandra 3-7 keV image of the source J004150.47-09. Panel (f): XMM-Newton/pn image of the source J011515.78+00. All images have been smoothed with a Gaussian with kernel radius 3.

those of $35 \mathrm{FRI}$ radio galaxies belonging to the $3 \mathrm{CR} / 3 \mathrm{CRR}$ catalogs and having X-ray data available. The $2-10 \mathrm{keV}$ luminosities of FRIs are from literature or obtained by a direct analysis of the data stored in the public archives (see Appendix B for details). Various samples of FRIs can be considered for the comparison with compact radio sources. For example the $3 \mathrm{C}$ sample includes $\sim 30 \mathrm{FRIs}$, the B2 sample is formed by $\sim 100$ radio galaxies, about half of them being FRIs. The recent FRICAT catalog is formed by 219 sources, selected from the SDSS/NVSS surveys, with a flux limit of $5 \mathrm{mJy}$ at $1.4 \mathrm{GHz}$. However, the multi-wavelength information is rather limited and, in particular, the coverage of X-ray observations is very small for all samples except for the 3C for which Chandra data are available for all sources up to $\mathrm{z}=1$ (Massaro et al. 2010, 2012, 2013). Being selected with a rather high flux threshold (9 Jy at $178 \mathrm{MHz}$ ), they represent the tip of the iceberg of the FRI population. While this means that the view of FRIs offered by the $3 \mathrm{C}$ sample is 
limited, they certainly provide us with a benchmark against which compare the properties of the compact radio sources.

The two distributions in Figure 4 (upper panel) clearly overlap: the two sample tests univariate program in the ASURV package (TWOST, Feigelson \& Nelson 1985; Isobe, Feigelson \& Nelson 1986) applied to the data (including upper limits) confirms their similarity $\left(\mathrm{P}_{\text {ASURV }}=0.76\right)^{12}$. We assume that $\mathrm{P}=0.05$ is the probability threshold to rule out the hypothesis that the two samples are drawn from the same parent population. This result indicates a strong correspondence between the X-ray cores of low-excitation FR0 and FRI radio galaxies ${ }^{13}$.

This point is strengthened by Figure 4 (lower panel) where the X-ray $(2-10 \mathrm{keV})$ and radio core $(5 \mathrm{GHz})$ luminosities of FR0s and FRIs are plotted together. Apart from the two objects having measurements at $4.9 \mathrm{GHz}$ (see Table 2), the luminosities at $5 \mathrm{GHz}$ of the compact sources were extrapolated from $1.4 \mathrm{GHz}$ (FIRST) data considering the radio spectral slopes reported in Table 2. The core luminosities of the extended radio galaxies are from literature (Buttiglione et al. 2010, 2011; Hardcastle et al. 2009). The two samples occupy the same area in the plot: the generalized Kendall's $\tau$ test (ASURV package: Isobe et al. 1986) gives a probability of correlation greater than $99.99 \%$ and 99.95\% for FRIs and FR0s (including upper limits), respectively.

We also tested the possible influence of redshift in driving this correlation estimating a partial rank coefficient ${ }^{14}$. The effect is negligible and the value of the correlation coefficient does not change significantly.

The correlation between $\mathrm{L}_{\mathrm{X}}-\mathrm{L}_{5 \mathrm{GHz}}$ already found for 3CR/FRIs (Balmaverde et al. 2006), it is now attested for FR0s pointing towards a jet origin of both the radio and $\mathrm{X}$-ray photons. As reported in Section 3.3, an intrinsic absorber is not required by the fit, at least for those sources having good quality X-ray spectra. Therefore, it is unlikely that the jet-related X-ray emission observed in FR0s is the unabsorbed component of a HEG-like spectrum. A similar result was obtained by Mingo et al. (2014), who concluded that the LEGs of their 2 Jy sample cannot be interpreted as simple heavily obscured HEGs (see also Baldi et al. 2010). This point is further strengthened below by the estimate of the Eddington-scaled luminosities for our FR0 sample.

From the stellar velocity dispersion relation of Tremaine et al. $(2002)^{15}$ we estimated the black hole masses $\left(\mathrm{M}_{\mathrm{BH}}\right)$ of our sample of FR0s. The values of $\mathrm{M}_{\mathrm{BH}}$ range between $\sim 10^{8}$ and $\sim 10^{9} \mathrm{M}_{\odot}$ (see Table 4 ). From the relation $\mathrm{L}_{\mathrm{bol}}=3500 \mathrm{~L}_{[\mathrm{OIII}]}$ (Heckman et al. 2004) we derived the bolometric luminosities and successively the Eddington-scaled luminosities ( $\dot{L}=\mathrm{L}_{\mathrm{bol}} / \mathrm{L}_{\mathrm{Edd}}$ ) given in Table 4 . These estimates for the FR0s of our sample correspond to low values

\footnotetext{
12 Probability value according to the Gehan's generalized Wilcoxon test.

13 A similar result is obtained even excluding upper limits and applying a Kolmogorov-Smirnov test to the data $\left(\mathrm{P}_{\mathrm{KS}}=0.45\right)$.

14 The partial rank coefficient estimates the correlation coefficient between two variables after removing the effect of a third. If

$\mathrm{A}$ and $\mathrm{B}$ are both related to the variable $\mathrm{z}$, the partial Kendall's $\tau$ correlation coefficient is: $\tau_{A B, z}=\frac{\tau_{A B}-\tau_{A z} \tau_{B z}}{\sqrt{\left(1-\tau_{A z}^{2}\right)\left(1-\tau_{B z}^{2}\right)}}$.

$15 \log \left(\mathrm{M}_{\mathrm{BH}} / \mathrm{M}_{\odot}\right)=(8.13 \pm 0.06)+(4.02 \pm 0.32) \log \left(\sigma / 200 \mathrm{~km} \mathrm{~s}^{-1}\right)$
}

of $\dot{L}\left(\sim 10^{-3}-10^{-5}\right)$ typical of inefficient accretion modes (ADAF-like, Narayan \& Yi 1994, 1995) and similar to those found for FRIs (see also Table B1) and for 2 Jy LEGs (Mingo et al. 2014).

This result strengthens the interpretation of a nonthermal origin of the high-energy nuclear emission in lowexcitation compact sources, already suggested by the correlation between radio and X-ray emissions.

\subsection{Compact radio galaxies versus BL Lac objects}

The radio compactness of FR0s is due to the lack of extended emission and it is not related to a Doppler boosting of the jet radiation. This is evident in the compact radio galaxies that show emission lines with large equivalent widths in their optical spectra (such as Tol1326-379, Grandi et al. 2016) but it is less straightforward in objects overwhelmed by the galaxy emission. Indeed, in this case, the stellar population dominates the emission hiding the nature of the underlying AGN that could be both a low-luminosity BL Lac with an extended jet pointed towards the observer, or a genuine compact radio galaxy.

In Figure 1 it is evident that FR0s and low-luminosity BL Lacs occupy different regions of the $\mathrm{L}_{[\mathrm{OIII}]}-\mathrm{L}_{1.4} \mathrm{GHz}$ plane. The two classes have similar emission-line luminosities but BL Lacs are more powerful in the radio band. This is expected since $\mathrm{L}_{[\mathrm{OIII}]}$ is an isotropic indicator of the AGN luminosity, while the radio emission suffers from relativistic effects.

The ratio between the $[\mathrm{OIII}] \lambda 5007$ line and the $2-10 \mathrm{keV}$ luminosities $\left(\mathrm{R}_{[\mathrm{OIII}]}=\mathrm{L}_{[\mathrm{OIII}]} / \mathrm{L}_{(2-10 \mathrm{keV})}\right)$ can be considered a useful tool to distinguish misaligned and aligned jets. We then collected [OIII] luminosities of FRIs and FR0s from literature (Buttiglione et al. 2010, 2011; Hardcastle et al. 2009; Leipski et al. 2009) and from SDSS/DR7 survey ${ }^{16}$, respectively (see Appendix B and Table 4). For the [OIII]line luminosity of BL Lacs we refer to the work of Capetti \& Raiteri (2015), while the X-ray 2-10 keV luminosity was directly obtained from the Swift/XRT instrument using the SSDC online data analysis tool.

The $\mathrm{R}_{[\mathrm{OIII}]}$ average values (including upper limits ${ }^{17}$ ) for FR0s and FRIs are consistent $\left(<\mathrm{R}_{[\mathrm{OIII}], \mathrm{FR} 0}>=-1.6 \pm 0.2\right.$ and $\left.<\mathrm{R}_{[\mathrm{OIII}], \mathrm{FRI}}>=-1.7 \pm 0.2\right)$. This is statistically attested by the TWOST test in ASURV (see Section 4.1 for details) that turns out with a probability $\mathrm{P}_{\mathrm{ASURV}}=0.9$. On the contrary, the Doppler boosting of the X-ray emission shifts the BL Lacs to lower values $<\mathrm{R}_{[\mathrm{OIII}], \text { BLLacs }}>=-3.3 \pm 0.2$.

\subsection{Compact radio galaxies versus young sources}

The comparison between FR0s and young radio sources in the X-ray band suffers from the paucity of dedicated studies in this field (Kunert-Bajraszewska et al. 2014 and references therein). The samples of young sources for which high-energy information are available include mainly powerful GPS and CSS. These sources are generally different from ours being characterized by high X-ray $(2-10 \mathrm{keV})$, radio

\footnotetext{
16 http://classic.sdss.org/dr7/

17 We used the Kaplan-Meier (KM) estimator in ASURV to derive the average values of $\mathrm{R}_{[\mathrm{OIII}]}$ in the presence of censored data.
} 
(5 GHz) and [OIII]-line luminosities typical of AGN with efficient accretion rates (Guainazzi et al. 2006; Vink et al. 2006; Labiano 2008; Siemiginowska et al.2008; Tengstrand et al. 2009). Moreover, 16 CSO sources recently studied by Siemiginowska et al. (2016) in X-rays showed spectra generally flat and absorbed by intrinsic column densities (see also Ostorero et al. 2017).

Our sources seem more similar to three low-luminosity compact sources (LLC; Kunert-Bajraszewska \& Thomasson 2009) discussed by Kunert-Bajraszewska et al. (2014) and classified as LEG. Their radio and X-ray luminosities (see Table 2 of their work) locate these sources in our correlation strip shown in Figure 4 (lower panel). The authors suggest that such LLC are intermittent radio sources rather than young objects evolving in FRIs, in line with the recent demographic study of Baldi et al. (2018a). They showed that the space density of FR0s in the local Universe $(\mathrm{z}<0.05)$ is larger by a factor of $\sim 5$ than FRIs, definitively rejecting the hypothesis that FR0s are young radio galaxies that will all eventually evolve into extended FRI radio sources.

\section{SUMMARY AND CONCLUSIONS}

We analyzed 19 FR0s selected according to the criteria discussed in Section 2 and having public X-ray observations. Most of the sources have short exposures and/or large offsets. In spite of the limited quality of the data, our analysis allowed us to characterize for the first time FR0 sources at high-energies.

FR0s have X-ray luminosities $(2-10 \mathrm{keV})$ between $10^{40}$. $10^{43} \mathrm{erg} \mathrm{s}^{-1}$, comparable to FRIs. The clear correlation between radio and X-ray luminosities observed in both compact and extended objects, favours the interpretation of a non-thermal origin of the $2-10 \mathrm{keV}$ photons. In agreement with FRIs, the high-energy emission in FR0s is produced by the jet. Moreover, the high black hole masses $\left(10^{8}-10^{9} \mathrm{M}_{\odot}\right)$ and the small values of the bolometric luminosities, as deduced from the [OIII]-emission line, suggest an inefficient accretion process (ADAF-like) at work also in the compact sources. These results confirm that the nuclear properties of FRIs and FR0s are similar and that the main difference between the two classes remains the lack of extended emission in FR0s.

We exclude important beaming effects in the X-ray spectra of FR0s on the basis of the ratio between the [OIII] $\lambda 5007$ line and the $2-10 \mathrm{keV}$ luminosity $\left(\mathrm{R}_{[\mathrm{OIII}]}\right)$. While the [OIII]-line luminosity is expected to be emitted isotropically, the X-ray radiation could be amplified by Doppler boosting effects. While FR0s and FRIs have similar $\mathrm{R}_{[\mathrm{OIII}]}$ $(\sim-1.7)$, low-luminosity BL Lacs, whose $\mathrm{X}$-ray radiation is beamed, have smaller values $\left(\mathrm{R}_{[\mathrm{OIII}]} \sim-3.3\right)$.

A comparison with the X-ray properties of young sources is limited by the paucity of X-ray studies of GPS, CSS and CSO. Considering the available data, we do not find spectral similarities between these sources and FR0s. Generally, the studied young sources have higher X-ray luminosities (they are probably associated with an efficient accretion disc) and often show signatures of intrinsic absorption. Therefore, FR0s could be different sources characterized by intermittent activity, as in the case of J004150.47-09 and/or
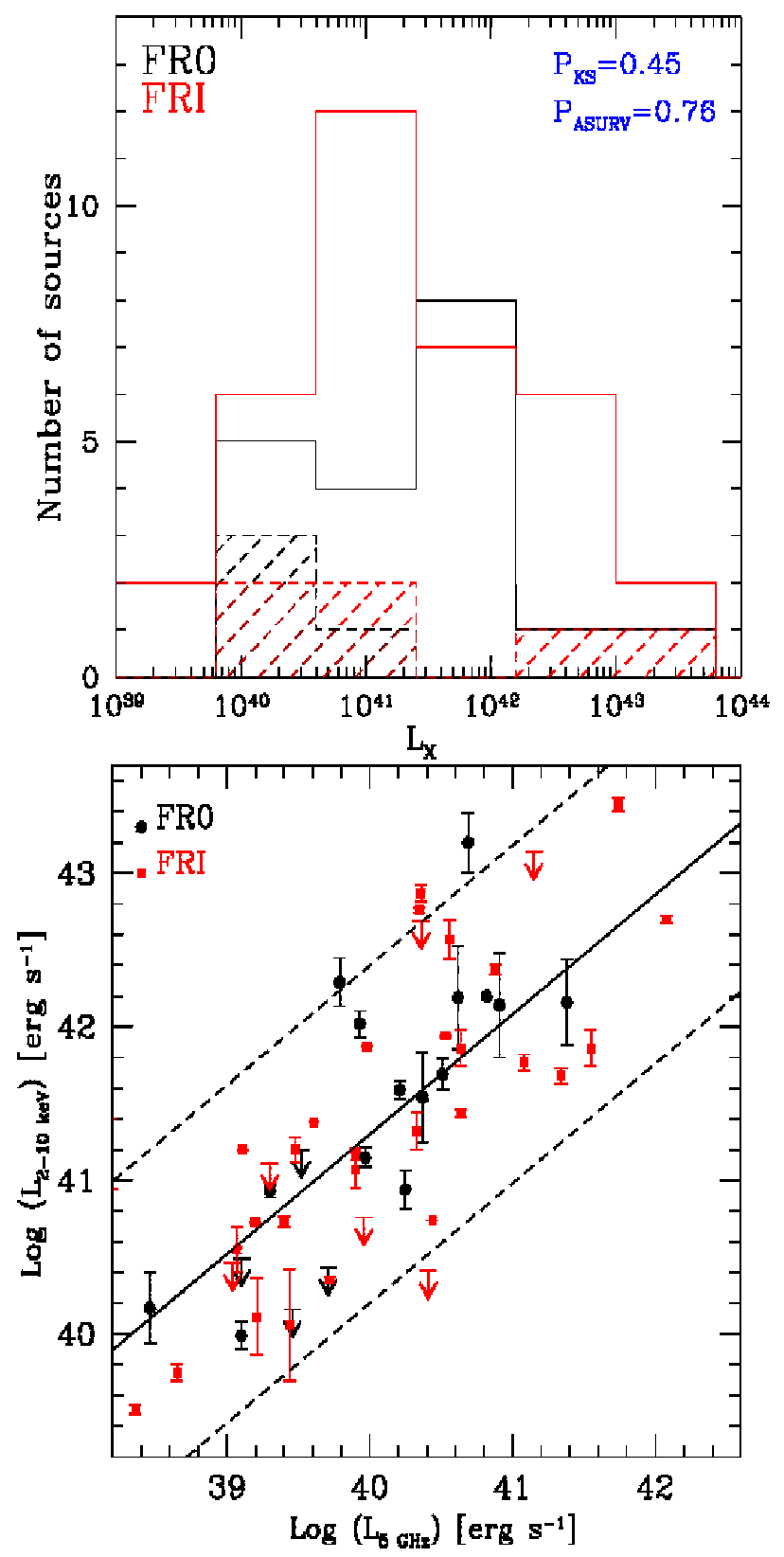

Figure 4. Upper panel: histogram of the X-ray luminosity for the FR0s of our sample (black solid line) and the comparative sample of FRIs (red solid line). No significant difference is observed between the two samples. The dashed histograms represent upper limits in each sample. In the plot the probabilities from the Kolmogorov-Smirnov test $\left(\mathrm{P}_{\mathrm{KS}}\right)$ and the TWOST test in ASURV (PASURV) are also reported. Lower panel: 2$10 \mathrm{keV}$ X-ray luminosity versus $5 \mathrm{GHz}$ radio core luminosity for FR0s (black circles) and FRIs (red squares). Arrows indicate upper limits. The black solid line is the linear regression for the overall sample of FR0s and FRIs (excluding the upper limits): $\log \mathrm{L}_{\mathrm{X}}=(7.8 \pm 0.6)+(0.8 \pm 0.1) \log \mathrm{L}_{5 \mathrm{GHz}}$ (see also Hardcastle et al. 2009). The black dashed lines are the uncertainties on the slope. 
by jets with intrinsic properties that prevent the formation and evolution of extended structures. 
Table 3. Main spectral parameters of the FR0 sample: (1) Name of the source, (2) redshift, (3) best-fit spectrum, (4) Galactic hydrogen column density, (5) power-law photon index, (6) temperature of the thermal component (APEC), (7) flux of the thermal component in the soft X-ray band (0.5-5 keV) corrected for Galactic absorption, (8) flux of the non-thermal component in the $2-10 \mathrm{keV}$ band corrected for Galactic absorption, (9) $\chi^{2} /$ degrees of freedom reported when the grouping was $\geq 15$. When the grouping was $<15$ the C-statistics was applied, (10) type of environment, (11) references for the environment.

\begin{tabular}{|c|c|c|c|c|c|c|c|c|c|c|}
\hline Source name & $\mathrm{z}$ & Best spectrum & $\begin{array}{c}\mathrm{N}_{H, \text { Gal }} \\
(\text { atoms cm } \\
-2\end{array}$ & $\Gamma$ & $\begin{array}{c}\mathrm{kT} \\
(\mathrm{keV})\end{array}$ & $\begin{array}{c}\mathrm{F}_{t h, 0.5-5 \mathrm{keV}} \\
\left(\mathrm{erg} \mathrm{cm}^{-2} \mathrm{~s}^{-1}\right)\end{array}$ & $\begin{array}{c}\mathrm{F}_{n u c, 2-10 \mathrm{keV}} \\
\left(\mathrm{erg} \mathrm{cm}^{-2} \mathrm{~s}^{-1}\right)\end{array}$ & $\chi^{2} /$ d.o.f. & Environment & Reference $^{d}$ \\
\hline J004150.47-09 & 0.055 & $\mathrm{PL}$ & $2.8 \times 10^{20}$ & 2.0 (fix) & - & - & $\left(4.5_{-2.8}^{+2.9}\right) \times 10^{-14}$ & $1.4 / 3$ & Cluster & 1 \\
\hline J010101.12-00 & 0.097 & PL & $3.2 \times 10^{20}$ & 2.0 (fix) & - & - & $<6.6 \times 10^{-15}$ & - & $?$ & - \\
\hline $\mathrm{J} 011515.78+00$ & 0.045 & $\mathrm{APEC}+\mathrm{PL}$ & $3.0 \times 10^{20}$ & $2.0 \pm 0.2$ & $0.8 \pm 0.1$ & $(1.5 \pm 0.4) \times 10^{-14}$ & $\left(2.9_{-0.4}^{+0.5}\right) \times 10^{-14}$ & $66 / 63$ & Cluster & 1 \\
\hline J015127.10-08 & 0.018 & $\mathrm{APEC}+\mathrm{PL}$ & $2.3 \times 10^{20}$ & 2.0 (fix) & $0.2_{-0.1}^{+0.3}$ & $\left(1.4_{-1.3}^{+113}\right) \times 10^{-13}$ & $<4.2 \times 10^{-14}$ & - & $?$ & - \\
\hline J080624.94+17 & 0.104 & PL & $3.3 \times 10^{20}$ & 2.0 (fix) & - & - & $\left(3.2_{-1.4}^{+1.7}\right) \times 10^{-13}$ & - & Cluster & 2 \\
\hline J092405.30+14 & 0.135 & $\mathrm{APEC}+\mathrm{PL}$ & $3.3 \times 10^{20}$ & $1.8 \pm 0.3$ & $1.3_{-0.6}^{+1.4}$ & $\left(5.9_{-5}^{+19}\right) \times 10^{-15}$ & $\left(3.2_{-2.8}^{+1.1}\right) \times 10^{-14}$ & $3 / 9$ & Cluster & 1 \\
\hline J093346.08+10 & 0.011 & $\mathrm{APEC}+\mathrm{PL}$ & $3.1 \times 10^{20}$ & $2.0($ fix $)$ & $<0.35$ & $<1.2 \times 10^{-13}$ & $\left(6.2_{-2.1}^{+2.7}\right) \times 10^{-14}$ & - & $\mathrm{CG}^{c}$ & 3 \\
\hline J094319.15+36 & 0.022 & PL & $1.1 \times 10^{20}$ & $2.3 \pm 0.4$ & - & - & $\left(7.9_{-2.0}^{+2.3}\right) \times 10^{-14}$ & - & $?$ & - \\
\hline $\mathrm{J} 104028.37+09$ & 0.019 & PL & $2.6 \times 10^{20}$ & $2.2 \pm 0.4$ & - & - & $(1.2 \pm 0.2) \times 10^{-14}$ & $3 / 7$ & Isolated & 4 \\
\hline $\mathrm{J} 114232.84+26$ & 0.03 & $\mathrm{APEC}+\mathrm{PL}$ & $2.0 \times 10^{20}$ & 2.0 (fix) & $0.67_{-0.06}^{+0.07}$ & $(4.5 \pm 0.1) \times 10^{-14}$ & $<1.3 \times 10^{-14}$ & - & $\mathrm{CG}^{c}$ & 3 \\
\hline $\mathrm{J} 115954.66+30$ & 0.106 & PL & $1.5 \times 10^{20}$ & 2.0 (fix) & - & - & $\left(4.9_{-3.1}^{+4.9}\right) \times 10^{-14}$ & - & $?$ & - \\
\hline $\mathrm{J} 122206.54+13$ & 0.081 & PL & $3.5 \times 10^{20}$ & 2.0 (fix) & - & - & $\left(1.0_{-0.7}^{+1.2}\right) \times 10^{-13}$ & - & Cluster & 5 \\
\hline $\mathrm{J} 125431.43+26$ & 0.069 & PL & $7.5 \times 10^{19}$ & $1.9 \pm 1.4$ & - & - & $\left(9.2_{-1.6}^{+1.8}\right) \times 10^{-14}$ & $4 / 5$ & $?$ & - \\
\hline Tol1326-379 & 0.028 & PL & $5.5 \times 10^{20}$ & $1.3 \pm 0.4$ & - & - & $\left(9.4_{-3.1}^{+1.9}\right) \times 10^{-13}$ & - & $?$ & - \\
\hline $\mathrm{J} 135908.74+28$ & 0.073 & $\mathrm{APEC}+\mathrm{PL}$ & $1.3 \times 10^{20}$ & 2.0 (fix) & $0.24(\mathrm{fix})^{a}$ & $\left(1.9_{-0.7}^{+0.8}\right) \times 10^{-13}$ & $\left(5.0_{-2.3}^{+2.2}\right) \times 10^{-14}$ & $0.5 / 2$ & Cluster & 1 \\
\hline J153901.66+35 & 0.078 & PL & $1.8 \times 10^{20}$ & 2.0 (fix) & - & - & $\left(1.1_{-1.0}^{+0.7}\right) \times 10^{-13}$ & - & $?$ & - \\
\hline $\mathrm{J} 160426.51+17$ & 0.041 & PL & $3.4 \times 10^{20}$ & $1.1 \pm 0.3$ & - & - & $\left(1.5_{-0.3}^{+0.5}\right) \times 10^{-13}$ & $2.5 / 8$ & Cluster & 1 \\
\hline $\mathrm{J} 171522.97+57$ & 0.027 & $\mathrm{APEC}+\mathrm{PL}$ & $2.2 \times 10^{20}$ & 2.0 (fix) & $1.1 \pm 0.1^{b}$ & $\left(1.2_{-0.5}^{+0.4}\right) \times 10^{-13}$ & $<1.9 \times 10^{-14}$ & $70 / 53$ & $\mathrm{CG}^{c}$ & 1 \\
\hline J235744.10-00 & 0.076 & $\mathrm{PL}$ & $3.3 \times 10^{20}$ & 2.0 (fix) & - & - & $\left(9.7_{-5.1}^{+7.9}\right) \times 10^{-14}$ & - & Isolated & 6 \\
\hline
\end{tabular}

${ }^{a}$ Due to the poor statistics of the fit this parameter is fixed to the best fit value.

${ }^{b}$ The Abundance parameter is left free and reaches a value of $\mathrm{Ab}=0.3_{-0.1}^{+0.3}$.

${ }^{c} \mathrm{CG}=$ compact group of galaxies.

${ }^{d}$ (1)-this work, (2)-Koester et al. (2007), (3)-Diaz-Gimenez et al. (2012), (4)-Colbert et al. (2001), (5)-Owen et al. (1995), (6)-Prada et al. (2003). 
Table 4. Radio, optical and X-ray properties of the FR0 sample. (1) Identity number, (2) nuclear radio luminosity at $5 \mathrm{GHz}$, (3) X-ray nuclear luminosity (2-10 keV) corrected for absorption, (4) [OIII] emission line luminosity. For all luminosities the proper k-correction was considered. (5) Estimated black hole masses for the sources of the sample, (6) Eddington-scaled luminosities $\left(\dot{L}=\mathrm{L}_{\mathrm{bol}} / \mathrm{L}_{\mathrm{Edd}}\right)$.

\begin{tabular}{lccccc}
\hline \hline Source name & $\begin{array}{c}\operatorname{LogL}_{5 \mathrm{GHz}} \\
\left(\mathrm{erg} \mathrm{s}^{-1}\right)\end{array}$ & $\begin{array}{c}\mathrm{LogL}_{\mathrm{X}, 2-10 \mathrm{keV}} \\
\left(\mathrm{erg} \mathrm{s}^{-1}\right)\end{array}$ & $\begin{array}{c}\mathrm{LogL}_{[\mathrm{OIII}]}{ }^{a} \\
\left(\mathrm{erg} \mathrm{s}^{-1}\right)\end{array}$ & $\begin{array}{c}\operatorname{LogM}_{B H} \\
\left(\mathrm{M}_{\odot}\right)\end{array}$ & $\dot{L}^{b}$ \\
\hline J004150.47-09 & 40.21 & 41.10 & 39.27 & 8.96 & $5.5 \times 10^{-5}$ \\
J010101.12-00 & 39.52 & $<41.20$ & 40.39 & 8.43 & $2.4 \times 10^{-3}$ \\
J011515.78+00 & 39.97 & 41.15 & 39.51 & 8.57 & $2.3 \times 10^{-4}$ \\
J015127.10-08 & 39.10 & $<40.49$ & 39.29 & 7.97 & $5.6 \times 10^{-4}$ \\
J080624.94+17 & 40.69 & 43.20 & 39.30 & 8.39 & $2.2 \times 10^{-4}$ \\
J092405.30+14 & 41.38 & 42.16 & 40.68 & 9.06 & $1.1 \times 10^{-3}$ \\
J093346.08+10 & 38.46 & 40.17 & 39.14 & 8.16 & $2.6 \times 10^{-4}$ \\
J094319.15+36 & 40.25 & 40.94 & 39.81 & 7.89 & $2.2 \times 10^{-3}$ \\
J104028.37+09 & 39.10 & 39.99 & 39.48 & 8.29 & $4.2 \times 10^{-4}$ \\
J115954.66+30 & 39.71 & $<40.43$ & 38.48 & 8.97 & $8.7 \times 10^{-6}$ \\
J114232.84+26 & 40.91 & 42.14 & 40.24 & 8.51 & $1.4 \times 10^{-3}$ \\
J122206.54+13 & 40.62 & 42.19 & 40.04 & 8.36 & $1.3 \times 10^{-3}$ \\
J125431.43+26 & 39.93 & 42.02 & 39.61 & 8.59 & $2.8 \times 10^{-4}$ \\
To11326-379 & 39.79 & 42.29 & 40.60 & 8.30 & $5.0 \times 10^{-3}$ \\
J135908.74+28 & 40.51 & 41.69 & 39.48 & 8.46 & $2.8 \times 10^{-4}$ \\
J153901.66+35 & 40.82 & 42.20 & 40.07 & 8.31 & $1.5 \times 10^{-3}$ \\
J160426.51+17 & 40.37 & 41.64 & 40.02 & 8.34 & $1.3 \times 10^{-3}$ \\
J171522.97+57 & 39.46 & $<40.16$ & 39.46 & 8.79 & $1.2 \times 10^{-4}$ \\
J235744.10-00 & 39.30 & 40.93 & 40.26 & 8.76 & $8.5 \times 10^{-4}$ \\
\hline
\end{tabular}

Data are provided by the SDSS Data Release 7 (http://www.sdss.org/)

$\dot{L}=\mathrm{L}_{\mathrm{bol}} / \mathrm{L}_{\mathrm{Edd}}$. The bolometric luminosity is derived using the relation

$\mathrm{L}_{\mathrm{bol}}=3500 \mathrm{~L}_{[\mathrm{OII}]}$ measured by Heckman et al. (2004). 


\section{ACKNOWLEDGEMENTS}

The authors thank the anonymous referee for his/her thoughtful comments that helped to improve the paper. ET acknowledges financial support from ASI-INAF grant 2015023-R.O. This work is based on data from the Chandra, $X M M-N e w t o n$ and Swift Data Archive. Part of this work is based on archival data, software or online services provided by the ASI Science Data Center (ASDC). We thank the Swift team for making the ToO observation of Tol1326379 possible. Funding for the SDSS and SDSS-II has been provided by the Alfred P. Sloan Foundation, the Participating Institutions, the National Science Foundation, the U.S. Department of Energy, the National Aeronautics and Space Administration, the Japanese Monbukagakusho, the Max Planck Society, and the Higher Education Funding Council for England. The SDSS Web Site is http://www.sdss.org/.

\section{REFERENCES}

Abdo, A.A., et al. 2010, ApJS, 188, 405

Ackermann, M., et al. 2015, ApJ, 810, 14

Baldi, R.D., Capetti, A. 2008, A\&A, 489, 989

Baldi, R.D., Capetti, A. 2009, A\&A, 508, 603

Baldi, R.D., Capetti, A. 2010, A\&A, 519, 48

Baldi, R.D., Capetti, A., Giovannini, G. 2015, A\&A, 576, A38

Baldi, R.D., Capetti, A., Massaro, F. 2018a, A\&A, 609, 1

Baldi, R.D., Williams, D.R.A., McHardy, I.M. et al. 2018b, arXiv:1802.02162

Balmaverde, B. \& Capetti, A. 2006, A\&A, 447, 97

Balmaverde, B., Capetti, A., Grandi, P. 2006, A\&A, 451, 35

Balmaverde, B. \& Capetti, A. 2015, A\&A, 581, 76

Becker, R.H., White, R.L. \& Helfand, D.J. 1995, ApJ, 450, 559

Bennett, A.S. 1962, MNRAS, 125, 75

Best, P.N., Kauffmann, G., et al. 2005, MNRAS, 362, 9

Best, P.N. \& Heckman, T.M. 2012, MNRAS, 421, 1569

Bharadwaj, V., Reiprich, T.H., Lovisari, L. \& Eckmiller, H.J. 2015, A\&A, 573, 75

Binette, L., Magris, C.G., Stasińska G., Bruzuel, A.G. 1994, A\&A, 292, 13

Bodo, G., Mamatsashvili, G., Rossi, P. \& Mignone, A. 2013, MNRAS, 434, 3030

Browne, I.W.A., Wilkinson, P.N., Jackson, N.J., et al. 2003, MNRAS, 341, 13

Buttiglione, S., Capetti, A., Celotti, A. et al. 2009, A\&A, 495, 1033

Buttiglione, S., Capetti, A., Celotti, A., et al. 2010, A\&A, 509, A6

Buttiglione, S., Capetti, A., Celotti, A., et al. 2011, A\&A, 525, 28

Cao, W., Rawlings, S. 2004, MNRAS, 349, 1419

Capetti, A., Baldi, R.D. 2011, A\&A, 529, 126

Capetti, A., Raiteri, C.M. 2015, A\&A, 580, A73

Capetti, A., Massaro, F., Baldi, R.D. 2017, A\&A, 598, A49

Chiaberge, M., Capetti, A., Celotti, A. 2002, A\&A, 394, 791

Colbert, J.W., Mulchaey, J.S., Zabludoff, A. 2001, AJ, 121, 808

Colla, G., Fanti, C., Fanti, R., et al. 1975, A\&A, 38, 209

Condon, J.J., Cotton, W.D., Greisen, E.W. et al. 1998, AJ, 115, 1693

Dasadia, S., Sun, M., Morandi, A. et al. 2016, MNRAS, 458, 681

de Vaucoulers, G., de Vaucoulers, A., and Corwin, H.G. 1976, Second Reference Catalogue of Bright Galaxies, Austin: University of Texas Press

Diaz-Gimenez, E., Mamon, G.A., Pacheco, M. et al. 2012, MNRAS, 426, 296

Dressler, A. 1980, ApJS, 42, 565
Edge, D.O., et al. 1959, MmRAS, 68, 37

Fabbiano, G., Kim, D.W., Trinchieri, G. 1992, ApJS, 80, 531

Fanti, R., Gioia, I., et al. 1978, A\&AS, 34, 341

Fanaroff, B.L., Riley, J.A. 1974, MNRAS, 167, 31

Feigelson, E.D. \& Nelson, P.I. 1985, ApJ, 293, 192

Feretti, L. \& Giovannini, G. 1994, A\&A, 281, 375

Ghisellini, G. 2011, AIP Conference Proceedings, 1381, 180

Giovannini, G., Feretti, L., et al. 1988, A\&A, 199, 73

Graham, A.W., Erwin, P., Caon, N., Trujillo, I. 2001, ApJ, 563, 11

Grandi, P., Capetti, A., Baldi, R.D. 2016, MNRAS, 457, 2

Griffith, M.R. et al. 1994, ApJS, 90, 179

Guainazzi, M., Siemiginowska, A., Stanghellini, C., et al. 2006, A\&A, 446, 87

Heckman, T.M., Kauffmann, G., Brinchmann, J. et al. 2004, ApJ, 613,109

Helfand, D.J., White, R.L. \& Becker, R.H. 2015, ApJ, 801, 26

Ho, L.C., Filippenko, A.V. \& Sargent, W.L.W. 1997, ApJS, 112, 315

Isobe, T., Feigelson, E.D. \& Nelson, P.I. 1986, ApJ, 306, 490

Jackson, N. \& Rawlings, S. 1997, MNRAS, 286, 241

Jackson, N., Battye, R.A., Browne, I.W.A. et al. 2007, MNRAS, 376,371

Kalberla, P.M.W., Burton, W.B., Hartmann, Dap, Arnal, E.M., Bajaja, E., Morras, R. \& Pöppel, W.G.L. 2005, A\&A, 440, 775

Kewley, L.J., Groves, B., Kauffmann, G. \& Heckman, T. 2006, MNRAS, 372, 961

Koester, B.P., McKay, T.A., Annis, J. et al. 2007, ApJ, 660, 239

Kunert-Bajraszewska, M., Thomasson, P. 2009, Astron. Nachr., 330,210

Kunert-Bajraszewska, M., Labiano, A., Siemiginowska, A. \& Guainazzi, M. 2014, MNRAS, 437, 3063

Hlavacek-Larrondo, J., Fabian, A. C., Edge, A. C., et al. 2013, MNRAS, 431, 1638

Hardcastle, M.J., Evans, D.A. and Croston, J.H. 2009, MNRAS, 396,1929

Laing, R.A., Jenkins, C.R., Wall, J.V. \& Unger, S.W. 1994, in The Physics of Active Galaxies, ed. G.V. Bicknell, M.A. Dopita \& P.J. Quinn, ASP Conf. Ser., 54, 201

Landt, H., Padovani, P., Perlman, E.S., Giommi, P. 2004, MNRAS, 351, 83

Lanz, L., Ogle, P.M., Evans, D., et al. 2015, ApJ, 801, 17

Leipski, C., Antonucci, R., Ogle, P., Whysong, D. 2009, ApJ, 701, 891

Marchesini, D., Celotti, A. \& Ferrarese, L. 2004, MNRAS, 351, 733

Massaro, F., Harris, D.E., Tremblay, G.R., et al. 2010, ApJ, 714, 589

Massaro, F., Tremblay, G.R., Harris, D.E., et al. 2012, ApJS, 203, 31

Massaro, F., Harris, D.E., Tremblay, G.R., et al. 2013, ApJS, 206,

Mezcua M. \& Prieto, M.A. 2014, ApJ, 787, 62

Mingo, B., Hardcastle, M.J., Croston, J.H., et al. 2014, MNRAS, 440,269

Mingo, B., Watson, M.G., Rosen, S.R., Hardcastle, M.J. et al. 2016, MNRAS, 462, 2631

Morganti, R., Killeen, N.E.B. \& Tadhunter, C.N. 1993, MNRAS, 263, 1093

Morganti, R., Oosterloo, T.A., Reynolds, J.E. et al. 1997, MNRAS, 284, 541

Murphy, T., Sadler, E.M., Ekers, R.D., et al. 2010, MNRAS, 402, 2403

Myers, S.T., Jackson, N.J., Browne, I.W.A., et al. 2003, MNRAS, 341, 1

Nagar, N.M., Falcke, H. \& Wilson, A.S. 2005, A\&A, 435, 521

Narayan, R. \& Yi, I. 1994, ApJ, 428, 13 
Narayan, R. \& Yi, I. 1995, ApJ, 444, 231

Nolan, P.L., et al. 2012, ApJS, 199, 31

Ogle, P., Boulanger, F., Guillard, P., et al. 2010, ApJ, 724, 1193

Ostorero, L., Morganti, R., Diaferio, A., et al. 2017, ApJ, 849, 34

Owen, F.N., Ledlow, M.J., Keel, W.C. 1995, AJ, 109, 14

Pandge, M.B., Vagshette, N.D., David, L.P., Patil, M.K. 2012, MNRAS, 421, 808

Pilkington, J.D.H., Scott, J.F. 1965, MmRAS, 69, 183

Prada, F., Vitvitska, M., Klypin, A., et al. 2003, ApJ, 598, 260

Rosen, S.R., Webb, N.A., Watson, M.G., et al. 2016, A\&A, 590, 1

Sadler, E.M., Ekers, R.D., Mahony, E.K., et al. 2014, MNRAS, 438, 796

Sadler, E.M. 2016, Astronomische Nachrichten, 337, 105

Sarzi, M., Shields, J.C., Schawinski, K. et al. 2010, MNRAS, 402, 2187

Spergel, D.N., et al. 2007, ApJS, 170, 377

Stickel, M., Fried, J.W., Kuehr, H. 1993, A\&AS, 98, 393

Tengstrand, O., Guainazzi, M., Siemiginowska, A., et al. 2009, A\&A, 501, 89

Tremaine, S., Gebhardt, K., Bender, R., et al. 2002, ApJ, 574, 740

Trichas, M., Green, P.J., Constantin, A. et al. 2013, ApJ, 778, 188

Ulvestad, J.S. \& Ho, L.C. 2001, ApJ, 558, 561

Vink, J., Snellen, I., Mack, K.-H. \& Schilizzi, R. 2006, MNRAS, 367,928

Woo, J.-H., Urry, M.C. 2002, ApJ, 579, 530

Yang, Y., Huo, Z., Xue, S., et al. 2004, ApJ, 614, 692

York, D.G., Adelman, J., Anderson, Jr., J.E., et al. 2000, AJ, 120, 1579

\section{Appendix}

\section{A NOTES AND ANALYSIS DETAILS ON INDIVIDUAL SOURCES}

\section{J004150.47-09}

At the centre of the cluster Abell 85. This cluster presents $\mathrm{X}$-ray cavities created by the central AGN, as it is evident from the Chandra 3-7 keV image (Hlavacek-Larrondo et al. 2013). The presence of such cavities suggests that this AGN produced in the past an extended radio source able to excavate the external medium; this is an indication of recurrency.

\section{J010101.12-00}

This source is part of the B15 sample (their ID590) observed with the JVLA. For this object we could only obtain an upper limit on the $2-10 \mathrm{keV}$ flux estimated from the Chandra count rate using WebPIMMS and assuming a spectral slope $\Gamma=2$.

\section{$\mathrm{J} 011515.78+00$}

This source, GIN061, is part of the sample of FR0s presented in B15 (their ID605). It lies at the outskirt of the Abell cluster A168 (Dressler 1980), as shown by the Chandra (Yang et al. 2004) and XMM-Newton (Figure 3) X-ray images. The good quality of the XMM-Newton spectrum allowed us to constrain the parameters of the power-law $(\Gamma=2.0 \pm 0.2)$ and the thermal component $(\mathrm{kT}=0.8 \pm 0.1)$.

\section{J015127.10-08}

The X-ray spectrum of this source (a.k.a. NGC 0707) can be reproduced by a power-law plus a thermal model with
$\mathrm{kT} \sim 0.2 \mathrm{keV}$. This latter component is dominant, and for the power-law we could estimate only an upper limit on the $2-10 \mathrm{keV}$ flux.

\section{J080624.94+17}

From the work of Koester et al. (2007) the source seems to reside in a galaxy cluster. However, the statistics of the $\mathrm{X}$-ray data is too low to constrain the parameters of a thermal component. Therefore the spectrum is fitted with an absorbed power-law fixing $\Gamma=2$.

\section{J092405.30+14}

The source resides close to the centre of the cluster Abell 795. The X-ray spectrum can be well reproduced by a power-law with $\Gamma=1.8$ plus an APEC component with $\mathrm{kT}=1.3 \mathrm{keV}$ related to the thermal gas of cluster.

\section{J093346.08+10}

In NGC 2911 an extended jet radio emission on subarcsecond scales has been observed (Mezcua \& Prieto 2014), meaning that this source is powerful enough to launch radio jets, at least on pc scales.

\section{J094319.15+36}

NGC 2965. The Swift spectrum of this source can be modeled with a steep power-law $(\Gamma=2.3)$ only absorbed by Galactic column density. However, the low statistics prevented us from establishing the presence of a possible thermal component.

\section{$\mathrm{J} 104028.37+09$}

NGC 3332 resides in an isolated elliptical galaxy (Colbert et al. 2001). Its X-ray spectrum can be reproduced by a steep $(\Gamma=2.2 \pm 0.4)$ power-law absorbed only by the Galactic column density.

\section{$\mathrm{J} 114232.84+26$}

NGC 3826 is a member of a compact group of galaxies included in the photometric catalog of Diaz-Gimenez et al. (2012). The source lies at the edge of the XMM-Newton/PN field of view (offset=13.7 arcmin) since the main target was the star GJ436. Inspite of the poor statistics, the spectrum is better fitted with two components: a thermal model, probably related to the ICM and a power-law emerging above $2 \mathrm{keV}$. When the spectral slope, initially fixed to $\Gamma=2$, was let free to vary it assumed a smaller value suggesting the presence of intrinsic absorption. We could not constrain the value of the intrinsic $\mathrm{N}_{\mathrm{H}}$ but providing an upper limit on this parameter $\mathrm{N}_{\mathrm{H}}<3.4 \times 10^{23} \mathrm{~cm}^{-2}$.

\section{$\mathrm{J} 115954.66+30$}

This source at $\mathrm{z}=0.106$ is among the three farthest objects of the sample. We do not have any information about the environment either from the Swift X-ray image or from the literature. We reproduced the XRT spectrum with a power-law fixing the photon index to $\Gamma=2$.

\section{J122206.54+13}

The source is also known with the name VPC0184 and is associated to the cluster Abell 1526, that however is not visible in our Swift/XRT image. The X-ray spectrum can be reproduced by a power-law with photon index fixed 
to a value of 2 . Indeed, the statistics is not good enough to constrain this parameter nor to establish the possible presence of thermal gas related to the cluster. For this reason we are aware that the X-ray flux that we report in Table 3 could be overestimated.

\section{$\mathrm{J} 125431.43+26$}

This source is already present in the Chandra Source Catalog to Sloan Digital Sky Survey sample (CSC/SDSS) by Trichas et al. (2013). The X-ray spectral parameters presented in this paper are in agreement with those found by Trichas et al. We have no information on the environment either from the X-ray image nor from the literature.

\section{Tol1326-379}

Tol1326-379 is a FR0 source at $\mathrm{z}=0.0284$ hosted in an early type galaxy. It is the first FR0 with an associated $\gamma$-ray counterpart (Grandi et al. 2106). Indeed, it is listed in the 3LAC catalog as 3FGLJ1330.0-3818 (Ackermann et al. 2015). As it is evident from Table 3 the photon index of this gamma-ray FR0 is quite flat $\Gamma=1.3 \pm 0.4$. However, the inclusion of an intrinsic absorber did not significantly improve the fit. We could only estimate an upper limit to the intrinsic column density $\mathrm{N}_{\mathrm{H}}<3.4 \times 10^{22} \mathrm{~cm}^{-2}$.

\section{J135908.74+28}

This source, Zw162-39, resides in the Abell cluster A1831 (Feretti \& Giovannini 1994), as it shown in Figure 3. Indeed, the residuals in the X-ray spectrum suggest the presence of a thermal component in addition to the power-law. The estimated best-fit value of the gas temperature is $\mathrm{kT}=0.24 \mathrm{keV}$.

\section{J153901.66+35}

The very low statistics of the X-ray spectrum prevents us from establishing the presence of diffuse emission around the source. Therefore the spectrum is fitted with an absorbed power-law fixing $\Gamma=2$.

\section{$\mathrm{J} 160426.51+17$}

This source, NGC $6040 \mathrm{~B}$, lies at the outskirt of the cluster Abell 2151 (a.k.a. Hercules cluster). Together with NGC 6040A, located at 0.5 arcmin, it forms an interacting pair (de Vaucoulers et al. 1976). The X-ray spectrum is well reproduced by an absorbed power-law. The flatness of the photon index ( $\Gamma=1.1$, see Table 3$)$ suggests the possible presence of extra absorption. However, the low statistics prevent us from constraining the value of this additional component, for which we could only estimate an upper limit $\mathrm{N}_{\mathrm{H}}<4.9 \times 10^{22} \mathrm{~cm}^{-2}$.

\section{$\mathrm{J} 171522.97+57$}

NGC 6338 is part of a cool-core cluster (Bharadwaj et al. 2015). The X-ray spectrum is dominated by the thermal component of the cluster, for which we could constrain also the abundances to a value of 0.3 . The power-law is marginally significant in fact we could only determine an upper limit to the flux of this component.

\section{J235744.10-00}

The X-ray flux of this source, that is part of the B15 sample (their ID535), is provided by the 3XMM-DR6 catalog
(Rosen et al. 2016). Prada et al. (2003) lists this source as an isolated galaxy.

\section{B FRI}

Radio, optical and X-ray data of the 35 3CR-3CRR/FRIs in Table B1 are from: Buttiglione et al. (2009, 2010, 2011), Leipski et al. (2009), Lanz et al. (2015), Balmaverde et al. (2006), Hardcastle et al. (2009), Ogle et al. (2010), Migliori et al. (2011), Mingo et al. (2014), Dasadia et al. (2016).

For eleven sources the X-ray luminosities were obtained directly analyzing Chandra data using the software CIAO (v.4.7) and calibration database v.4.6.9. We followed standard procedures to extract source and background spectra. For the source we chose a circular region varying between 2.5" and 7". Only in two cases, e.g. 3C 371 and 3C 465, data were strongly piled up and therefore the spectra were extracted from annular regions $\left(\mathrm{r}_{i n}=0.5 ", \mathrm{r}_{\text {out }}=2.5 "\right)$. All background spectra were taken from annular regions with $\mathrm{r}_{\text {in }}$ and $\mathrm{r}_{\text {out }}$ ranging between 3 "-16" and 4"-30", respectively. Data were then grouped to a minimum of 15 counts per bin over the energy range $0.5-7 \mathrm{keV}$. This allows us to apply the $\chi^{2}$ statistics. For 3C 29, 3C 76.1 and 3C 129 data were not grouped and the C-statistics was applied. As a baseline model we adopted a power-law absorbed by Galactic column density. The photon index was let free to vary except for 3C 310 and $3 \mathrm{C} 424$ : in these cases $\Gamma$ was fixed to 2 . When the residuals were not satisfactory a thermal component was added to the data. Finally, in case the value of the photon index was very flat, we included an intrinsic $\mathrm{N}_{H}$ to the model. The results of the spectral analysis are summarized in Table B2. 
Table A1. Details of the data reduction for the sample of FR0s.

\begin{tabular}{|c|c|c|c|c|c|c|}
\hline $\begin{array}{l}\text { Source } \\
\text { (Instr.) }\end{array}$ & Reg. & Shape $^{a}$ & $\begin{array}{l}\mathbf{R}^{b} \\
{\left[{ }^{\prime \prime}\right]}\end{array}$ & $\begin{array}{l}\mathbf{R}_{i n}{ }^{c} \\
{\left[{ }^{\prime \prime}\right]}\end{array}$ & $\begin{array}{l}\mathbf{R}_{\text {out }}{ }^{d} \\
{\left[{ }^{\prime \prime}\right]}\end{array}$ & $\begin{array}{l}\text { X-ray } \\
\text { cluster }^{e}\end{array}$ \\
\hline $\begin{array}{l}\text { J004150.47-09 } \\
\text { (Chandra) }\end{array}$ & $\begin{array}{l}\mathrm{s} \\
\mathrm{b}\end{array}$ & $\begin{array}{l}\mathrm{c} \\
\mathrm{a}\end{array}$ & $\begin{array}{l}2.8 \\
-\end{array}$ & $-\overline{5}$ & $\begin{array}{l}- \\
7.8\end{array}$ & Yes \\
\hline $\begin{array}{l}\text { J010101.12-00 } \\
\text { (Chandra) }\end{array}$ & $\begin{array}{l}\mathrm{s} \\
\mathrm{b}\end{array}$ & $\begin{array}{l}\mathrm{c} \\
\mathrm{a}\end{array}$ & $\begin{array}{l}5 \\
-\end{array}$ & - & $\begin{array}{l}- \\
9.6\end{array}$ & $?$ \\
\hline $\begin{array}{l}\text { J011515.78+00 } \\
(\mathrm{XMM})\end{array}$ & $\begin{array}{l}\mathrm{s} \\
\mathrm{b}\end{array}$ & $\begin{array}{l}\mathrm{c} \\
\mathrm{c}\end{array}$ & $\begin{array}{l}16 \\
16 \\
\end{array}$ & - & - & Yes \\
\hline $\begin{array}{l}\text { J015127.10-08 } \\
\text { (Swift) }\end{array}$ & $\begin{array}{l}\mathrm{s} \\
\mathrm{b}\end{array}$ & $\begin{array}{l}\mathrm{c} \\
\mathrm{a}\end{array}$ & $\begin{array}{l}20 \\
-\end{array}$ & $\begin{array}{l}- \\
40\end{array}$ & - & $?$ \\
\hline $\begin{array}{l}\text { J080624.94+17 } \\
\text { (Swift) }\end{array}$ & $\begin{array}{l}\mathrm{s} \\
\mathrm{b}\end{array}$ & $\begin{array}{l}\mathrm{c} \\
\mathrm{a}\end{array}$ & $\begin{array}{l}20 \\
-\end{array}$ & $\begin{array}{l}- \\
40\end{array}$ & - & $?$ \\
\hline $\begin{array}{l}\text { J092405.30+14 } \\
\text { (Chandra) }\end{array}$ & $\begin{array}{l}\mathrm{s} \\
\mathrm{b}\end{array}$ & $\begin{array}{l}\mathrm{c} \\
\mathrm{a} \\
\end{array}$ & $\begin{array}{l}2.5 \\
-\end{array}$ & $\begin{array}{l}- \\
3.8 \\
\end{array}$ & 5.4 & Yes \\
\hline $\begin{array}{l}\text { J093346.08+10 } \\
\text { (Swift) }\end{array}$ & $\begin{array}{l}\mathrm{s} \\
\mathrm{b}\end{array}$ & $\begin{array}{l}\mathrm{c} \\
\mathrm{a}\end{array}$ & $\begin{array}{l}20 \\
-\end{array}$ & $\begin{array}{l}- \\
40\end{array}$ & - & $?$ \\
\hline $\begin{array}{l}\text { J094319.15+36 } \\
\text { (Swift) }\end{array}$ & $\begin{array}{l}\mathrm{s} \\
\mathrm{b}\end{array}$ & $\begin{array}{l}\mathrm{c} \\
\mathrm{a}\end{array}$ & $\begin{array}{l}20 \\
-\end{array}$ & - & $\begin{array}{l}- \\
80\end{array}$ & $?$ \\
\hline $\begin{array}{l}\text { J104028.37+09 } \\
\text { (XMM) }\end{array}$ & $\begin{array}{l}\mathrm{s} \\
\mathrm{b}\end{array}$ & $\begin{array}{l}\mathrm{c} \\
\mathrm{c}\end{array}$ & $\begin{array}{l}22 \\
22\end{array}$ & $\begin{array}{l}- \\
-\end{array}$ & $\begin{array}{l}- \\
-\end{array}$ & No \\
\hline $\begin{array}{l}\text { J114232.84+26 } \\
\text { (XMM) }\end{array}$ & $\begin{array}{l}\mathrm{s} \\
\mathrm{b}\end{array}$ & $\begin{array}{l}\mathrm{c} \\
\mathrm{a}\end{array}$ & $\begin{array}{l}26.5 \\
- \\
\end{array}$ & $\begin{array}{l}- \\
57.4\end{array}$ & $\begin{array}{l}- \\
87.7\end{array}$ & $?$ \\
\hline $\begin{array}{l}\text { J115954.66+30 } \\
\text { (Swift) }\end{array}$ & $\begin{array}{l}\mathrm{s} \\
\mathrm{b}\end{array}$ & $\begin{array}{l}\mathrm{c} \\
\mathrm{a}\end{array}$ & $\begin{array}{l}20 \\
-\end{array}$ & $\begin{array}{l}- \\
40\end{array}$ & - & $?$ \\
\hline $\begin{array}{l}\text { J122206.54+13 } \\
\text { (Swift) }\end{array}$ & $\begin{array}{l}\mathrm{s} \\
\mathrm{b}\end{array}$ & $\begin{array}{l}\mathrm{c} \\
\mathrm{a}\end{array}$ & $\begin{array}{l}20 \\
-\end{array}$ & $\begin{array}{l}- \\
40\end{array}$ & - & $?$ \\
\hline $\begin{array}{l}\text { J125431.43+26 } \\
\text { (Chandra) }\end{array}$ & $\begin{array}{l}\mathrm{s} \\
\mathrm{b}\end{array}$ & $\begin{array}{l}\mathrm{c} \\
\mathrm{a}\end{array}$ & $\begin{array}{l}2.7 \\
-\end{array}$ & - & - & No \\
\hline $\begin{array}{l}\text { Tol1326-379 } \\
\text { (Swift) }\end{array}$ & $\begin{array}{l}\mathrm{s} \\
\mathrm{b}\end{array}$ & $\begin{array}{l}\mathrm{c} \\
\mathrm{a}\end{array}$ & $\begin{array}{l}20 \\
-\end{array}$ & $\begin{array}{l}- \\
40\end{array}$ & - & No \\
\hline $\begin{array}{l}\text { J135908.74+28 } \\
\text { (Chandra) }\end{array}$ & $\begin{array}{l}\mathrm{s} \\
\mathrm{b}\end{array}$ & $\begin{array}{l}\mathrm{c} \\
\mathrm{a}\end{array}$ & $\begin{array}{l}4.5 \\
-\end{array}$ & $-\overline{8}$ & $\begin{array}{l}- \\
16.7\end{array}$ & Yes \\
\hline $\begin{array}{l}\text { J153901.66+35 } \\
\text { (Swift) }\end{array}$ & $\begin{array}{l}\mathrm{s} \\
\mathrm{b}\end{array}$ & $\begin{array}{l}\mathrm{c} \\
\mathrm{a}\end{array}$ & $\begin{array}{l}20 \\
-\end{array}$ & $\begin{array}{l}- \\
40\end{array}$ & - & $?$ \\
\hline $\begin{array}{l}\text { J160426.51+17 } \\
\text { (Chandra) }\end{array}$ & $\begin{array}{l}\mathrm{s} \\
\mathrm{b}\end{array}$ & $\begin{array}{l}\mathrm{c} \\
\mathrm{a}\end{array}$ & $\begin{array}{l}6.6 \\
-\end{array}$ & -8 & $\begin{array}{l}- \\
14.5\end{array}$ & Yes \\
\hline $\begin{array}{l}\text { J171522.97+57 } \\
\text { (Chandra) }\end{array}$ & $\begin{array}{l}\mathrm{s} \\
\mathrm{b}\end{array}$ & $\begin{array}{l}\mathrm{c} \\
\mathrm{a}\end{array}$ & $\begin{array}{l}3.3 \\
-\end{array}$ & $\begin{array}{l}- \\
4.7\end{array}$ & $\begin{array}{l}- \\
9.5\end{array}$ & Yes \\
\hline $\mathrm{J} 235744.10-00^{f}$ & - & - & - & - & - & $?$ \\
\hline
\end{tabular}

${ }^{a}$ Shape of the extraction region: $\mathrm{c}=$ circle, $\mathrm{a}=$ annulus.

${ }^{b}$ Radius of the circular region.

${ }^{c}$ Internal radius of the annular region.

${ }^{d}$ External radius of the annular region.

${ }^{e}$ The source is within (or at the edge of) a cluster or a group of galaxies in the X-ray image.

$f$ The source is part of the 3XMM-DR6 catalog of serendipitous sources (Rosen et al. 2016). 
Table B1. Multi-wavelength properties of the comparison sample of FRIs: (1) name, (2) redshift, (3) radio core luminosity at 5 GHz, (4) X-ray (2-10 keV) luminosity, (5) [OIII] emission line luminosity, (6) NVSS 1.4 GHz radio luminosity, (7) black hole masses from Marchesini et al. (2004), Woo \& Urry (2002), Graham et al. (2001), Cao \& Rawlings (2004),

(8) Eddington-scaled luminosities $(\dot{L}),(9)$ references for the X-ray luminosities. For all luminosities the proper k-correction was considered.

\begin{tabular}{|c|c|c|c|c|c|c|c|c|}
\hline Name & $\mathrm{z}$ & $\log \mathrm{L}_{(5 \mathrm{GHz})}$ & $\log \mathrm{L}_{(2-10 \mathrm{keV})}$ & $\log \mathrm{L}_{[\mathrm{OIII}]}$ & $\log \mathrm{L}_{(1.4 \mathrm{GHz})}$ & $\mathrm{M}_{\mathrm{BH}}$ & $\dot{L}^{a}$ & Ref. \\
\hline $3 \mathrm{C} 29$ & 0.0448 & 40.33 & 41.38 & 40.09 & 41.32 & 8.81 & $5.1 \times 10^{-4}$ & 2 \\
\hline $3 \mathrm{C} 31$ & 0.0169 & 39.4 & 40.73 & 39.46 & 39.90 & 7.89 & $1.0 \times 10^{-3}$ & 1 \\
\hline $3 \mathrm{C} 66 \mathrm{~B}$ & 0.0215 & 39.9 & 41.17 & 40.05 & 40.32 & 8.84 & $4.4 \times 10^{-4}$ & 1 \\
\hline $3 \mathrm{C} 75$ & 0.0232 & 39.3 & $<41.11$ & $<39.92$ & 37.86 & 9.0 & $<2.2 \times 10^{-4}$ & 1 \\
\hline 3C 76.1 & 0.0324 & 39.07 & 40.92 & 39.85 & 40.74 & 8.13 & $1.4 \times 10^{-3}$ & 2 \\
\hline $3 \mathrm{C} 78$ & 0.0288 & 40.88 & 42.37 & 39.41 & 41.17 & 8.98 & $7.2 \times 10^{-5}$ & 1 \\
\hline 3C 83.1 & 0.0251 & 39.11 & 41.2 & $<39.5$ & 40.40 & 9.01 & $<8.3 \times 10^{-5}$ & 1 \\
\hline $3 \mathrm{C} 84$ & 0.0176 & 42.08 & 42.7 & 41.6 & 41.33 & 9.28 & $5.6 \times 10^{-3}$ & 1 \\
\hline $3 \mathrm{C} 89$ & 0.138 & 41.08 & 41.77 & 40.51 & 42.09 & 8.83 & $1.3 \times 10^{-3}$ & 5 \\
\hline $3 \mathrm{C} 129$ & 0.0208 & 39.21 & 40.11 & $<39.85$ & 40.74 & - & - & 2 \\
\hline $3 \mathrm{C} 130$ & 0.109 & 40.64 & 41.57 & - & 41.62 & - & - & 2 \\
\hline 3C 189 & 0.043 & 40.53 & 41.94 & 39.94 & 41.20 & 8.93 & $2.7 \times 10^{-4}$ & 1 \\
\hline $3 \mathrm{C} 264$ & 0.0217 & 39.98 & 41.87 & 39.2 & 40.71 & 8.85 & $6.0 \times 10^{-5}$ & 3 \\
\hline $3 \mathrm{C} 270$ & 0.0074 & 39.2 & 40.73 & 38.96 & 39.83 & 8.57 & $6.6 \times 10^{-5}$ & 1 \\
\hline $3 \mathrm{C} 272.1$ & 0.0037 & 38.36 & 39.51 & 38.2 & 39.12 & 8.35 & $1.9 \times 10^{-5}$ & 1 \\
\hline $3 \mathrm{C} 274$ & 0.0037 & 39.72 & 40.35 & 38.99 & 40.76 & 8.26 & $1.4 \times 10^{-4}$ & 1 \\
\hline $3 \mathrm{C} 293$ & 0.0452 & 40.36 & 42.78 & 39.8 & 41.42 & 7.99 & $1.7 \times 10^{-3}$ & 3 \\
\hline $3 \mathrm{C} 296$ & 0.0237 & 39.61 & 41.38 & 39.78 & 40.39 & 9.13 & $1.2 \times 10^{-4}$ & 1 \\
\hline $3 \mathrm{C} 310$ & 0.053 & 40.41 & $<40.41$ & 40.05 & 41.86 & 8.29 & $1.5 \times 10^{-3}$ & 2 \\
\hline 3 C 315 & 0.1083 & 41.34 & 41.68 & 40.86 & 42.17 & 8.7 & $3.9 \times 10^{-3}$ & 4 \\
\hline $3 \mathrm{C} 317$ & 0.0342 & 40.64 & 41.44 & 40.35 & 41.30 & 8.80 & $9.5 \times 10^{-4}$ & 1 \\
\hline 3C 338 & 0.0303 & 39.96 & $<40.76$ & 39.57 & 41.02 & 9.23 & $5.9 \times 10^{-5}$ & 1 \\
\hline 3C 346 & 0.162 & 41.74 & 43.45 & 41.32 & 42.53 & 8.89 & $7.2 \times 10^{-3}$ & 1 \\
\hline 3C 348 & 0.154 & 40.36 & $<42.69$ & 40.4 & 41.85 & 8.84 & $9.8 \times 10^{-4}$ & 1 \\
\hline 3C 371 & 0.05 & 41.55 & 42.89 & 40.94 & 41.17 & - & - & 2 \\
\hline 3C 386 & 0.017 & 38.65 & 39.75 & 40.2 & 40.62 & 8.5 & $1.3 \times 10^{-3}$ & 4 \\
\hline $3 \mathrm{C} 402$ & 0.0259 & 39.48 & 41.20 & $<39.42$ & 40.60 & 8.18 & $<4.7 \times 10^{-4}$ & 2 \\
\hline $3 \mathrm{C} 424$ & 0.126 & 40.56 & 42.57 & 40.80 & 42.15 & 8.17 & $1.1 \times 10^{-2}$ & 2 \\
\hline $3 \mathrm{C} 438$ & 0.29 & 41.15 & $<43.14$ & 41.46 & 43.01 & 8.80 & $1.2 \times 10^{-2}$ & 3 \\
\hline $3 \mathrm{C} 442$ & 0.0263 & 38.19 & 41.17 & 39.21 & 40.22 & 8.28 & $2.3 \times 10^{-4}$ & 2 \\
\hline $3 \mathrm{C} 449$ & 0.0181 & 39.04 & $<40.46$ & 39.19 & 39.32 & 7.71 & $8.1 \times 10^{-4}$ & 1 \\
\hline 3C 465 & 0.0303 & 40.44 & 40.60 & 39.81 & 40.43 & 9.32 & $8.3 \times 10^{-5}$ & 2 \\
\hline NGC 6109 & 0.0296 & 39.44 & 40.06 & - & 40.01 & - & - & 3 \\
\hline NGC 6251 & 0.024 & 40.35 & 42.76 & 39.86 & 40.15 & 8.77 & $3.3 \times 10^{-4}$ & 6 \\
\hline NGC 7385 & 0.026 & 39.90 & 41.07 & - & 40.41 & - & - & 2 \\
\hline
\end{tabular}

${ }^{a} \dot{L}=\mathrm{L}_{\mathrm{bol}} / \mathrm{L}_{\mathrm{Edd}}$. The bolometric luminosity is derived from the relation $\mathrm{L}_{\mathrm{bol}}=3500 \mathrm{~L}_{[\mathrm{OIII}]}$

(Heckman et al. 2004) as for FR0s (see Table 4).

(1)-Balmaverde et al. (2006), (2)-this work, (3)-Hardcastle et al. (2009), (4)-Ogle et al. (2010)

(5)-Dasadia et al. (2016), (6)-Migliori et al. (2011) 


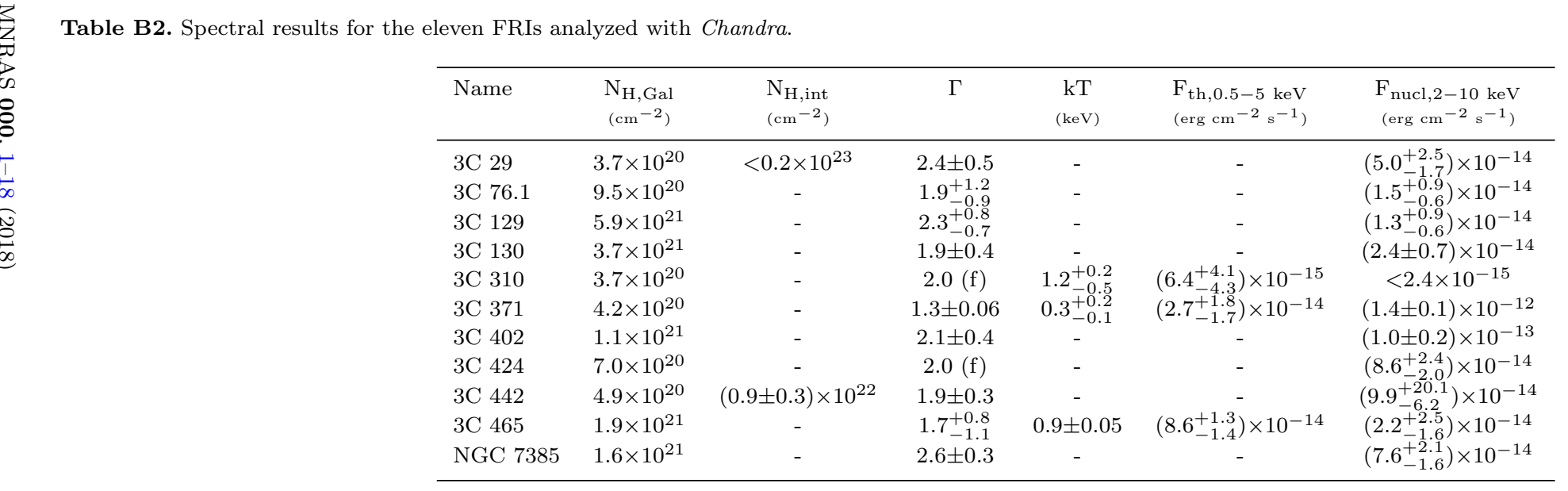




\section{E. Torresi et al.}

This paper has been typeset from a $\mathrm{T}_{\mathrm{E}} \mathrm{X} / \mathrm{LAT}_{\mathrm{E}} \mathrm{X}$ file prepared by the author. 